Pacific Journal of Mathematics

LIFTING UNITS IN SELF-INJECTIVE RINGS AND AN INDEX 


\title{
LIFTING UNITS IN SELF-INJECTIVE RINGS AND AN INDEX THEORY FOR RICKART $C^{*}$-ALGEBRAS
}

\author{
Pere Menal and Jaume Moncasi
}

In this paper we study the following question: If $R$ is $a$ right self-injective ring and $I$ an ideal of $R$, when can the units of $R / I$ be lifted to units of $R$ ?

We answer this question in terms of $K_{0}(I)$. For a purely infinite regular right self-injective ring $R$ we obtain an isomorphism between $K_{1}(R / I)$ and $K_{0}(I)$ which can be viewed as an analogue of the index map for Fredholm operators.

By giving a purely algebraic description of the connecting map $K_{1}(A / I) \rightarrow K_{0}(I)$ in the case where $A$ is a Rickart $C^{*}$-algebra, we are able to extend the classical index theory to Rickart $C^{*}$-algebras in a way which also includes Breuer's theory for $W^{*}$-algebras.

0. Preliminary results. Throughout this paper $R$ will denote an associative ring with 1 . By a $r n g$ we mean a ring which does not necessarily have a 1 .

We write $M_{n}(R)$ for the ring of all $n \times n$ matrices over $R$, and $\mathrm{GL}_{n}(R)$ for the group of units of $M_{n}(R)$, though we shall write $U(R)$ rather than $\mathrm{GL}_{1}(R)$. For $1 \leq i, j \leq n$ let $e_{i j} \in M_{n}(R)$ be the usual matrix units. Define $E_{n}(R)$ to be the subgroup of $\mathrm{GL}_{n}(R)$ generated by all the matrices of the form $1+r e_{i j}, r \in R, i \neq j$; and $G E_{n}(R)$ to be the subgroup of $\mathrm{GL}_{n}(R)$ generated by $E_{n}(R)$ together with the subgroup $D_{n}(R)$ of all invertible diagonal matrices. If $G E_{n}(R)=\operatorname{GL}_{n}(R)$, then we say that $R$ is a $G E_{n}-$ ring; if $R$ is a $G E_{n}$-ring for all $n>1$ then $R$ is said to be a $G E$-ring.

If $R$ is a $G E_{n}$-ring, then $E_{n}(R)$ is a normal subgroup of $\mathrm{GL}_{n}(R)$ and hence $\mathrm{GL}_{n}(R)=D_{n}(R) E_{n}(R)$.

Let $\operatorname{GL}(R)$ denote the direct limit of the directed system

$$
U(R) \rightarrow \mathrm{GL}_{2}(R) \rightarrow \mathrm{GL}_{3}(R) \rightarrow \cdots
$$

where each $a \in \mathrm{GL}_{n}(R)$ is mapped to

$$
\left(\begin{array}{ll}
a & 0 \\
0 & 1
\end{array}\right)
$$


in $\mathrm{GL}_{n+1}(R)$. Then $K_{1}(R)$ is defined to be $\mathrm{GL}(R)^{\mathrm{ab}}$, that is $\mathrm{GL}(R)$ abelianized.

Note that the canonical map $U(R) \rightarrow K_{1}(R)$ is onto in the case where $R$ is a $G E$-ring.

Let $I$ be a rng and $R$ a ring containing $I$ as an ideal. Let $P(I)$ denote the class of all finitely generated projective right $R$-modules $A$ such that $A I=A$. We say that $A, B \in P(I)$ are equivalent if $A \oplus C \approx$ $B \oplus C$ for some $C \in P(I)$. Denote by [A] the equivalence class of $A \in P(I)$. Thus the set $\{[A] \mid A \in P(I)\}$ with the operation $[A]+[B]=$ $[A \oplus B]$ is a cancellative abelian semigroup. We write $G(I)$ for its associated universal abelian group. Then every element of $G(I)$ has the form $[A]-[B]$ for suitable $A, \quad B \in P(I)$ and $[A]-[B]=$ [ $\left.A^{\prime}\right]-\left[B^{\prime}\right]$ if and only if $A \oplus B^{\prime} \oplus C \approx A^{\prime} \oplus B \oplus C$ for some $C \in P(I)$. It is not difficult to show that $P(I)$ consists of all $R$-modules $A$ such that $A \approx e\left(R^{n}\right)$ for some idempotent $n \times n$ matrix $e$ with entries in $I$. Thus, we see that $G(I)$ depends only on the structure of the rng $I$ and not on the involving ring $R$. Note that $G$ is a functor from the category of rngs into the category of abelian groups such that preserves direct limits.

For a ring $R, G(R)$ is simply $K_{0}(R)$. Recall that Bass and Milnor have defined a functor $K_{0}$ on the category of rngs; following Milnor [14, $\S 4$ ], we consider any ring $R$ containing $I$ as an ideal, let $\pi: R \rightarrow R / I$ be the natural surjection, and form the pullback

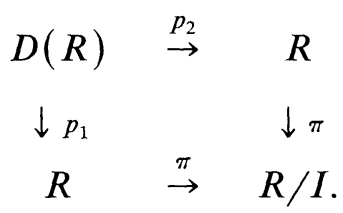

Then $K_{0}(I, R)$ is defined as the kernel of $K_{0}\left(p_{1}\right): K_{0}(D(R)) \rightarrow$ $K_{0}(R)$. In [2] it is proved that $K_{0}(I, R)$ depends only on $I$. Furthermore, there is an exact sequence, $\mathrm{cf} .[\mathbf{1 4}, \S 4]$ :

$$
K_{1}(R) \rightarrow K_{1}(R / I) \stackrel{\delta}{\rightarrow} K_{0}(I, R) \rightarrow K_{0}(R) \rightarrow K_{0}(R / I) .
$$

Let $I$ be a rng that is an $F$-algebra, where $F$ is either $\mathbf{Z}$ or a commutative field. Consider $I^{1}=I \oplus F$, the unitification of $I$ by $F$; by applying the above exact sequence we obtain

$$
K_{0}\left(I, I^{1}\right)=\operatorname{Ker}\left(K_{0}\left(I^{1}\right) \rightarrow K_{0}(F)\right) .
$$

When we write $K_{0}(I)$ we will have $K_{0}\left(I, I^{1}\right)$ in mind. 
If $I$ is a ring with unit $e$, then there is a ring decomposition $I^{1}=I \times(1-e) F$. Therefore $K_{0}\left(I^{1}\right)=K_{0}(I) \oplus K_{0}(F)$ and so $K_{0}\left(I, I^{1}\right)$ $=K_{0}(I)$. Hence we see that $K_{0}(I)$ agrees with the corresponding $K_{0}$ of $I$, where $I$ is viewed as a ring.

Let $I$ be a rng. With each $A \in P(I)$ we can associate its class in $K_{0}(I)$. In this way we obtain a group homomorphism $\phi: G(I) \rightarrow K_{0}(I)$. In the case where $\phi$ is an isomorphism we shall write $G(I)=K_{0}(I)$. When this occurs there is a very simple form for the elements in $K_{0}(I, R)$. More precisely, if $A \in P(I)$, then $0 \times A$ is a projective $D(R)$-module, and one easily obtains a group isomorphism

$$
K_{0}(I)=G(I) \rightarrow K_{0}(I, R)
$$

in which $[A] \mapsto[0 \times A]$.

In general we do not know whether $K_{0}(I)=G(I)$ but the following easy result will be enough for our purposes.

Proposition 0.1. Let I be an ideal of an F-algebra $R$, where $F$ is either $\mathbf{Z}$ or a commutative field. Suppose there exists a set $E$ of idempotents of $I$ such that for each pair $e, f \in E$ there exists $g \in E$ such that $e R e+f R f \subseteq$ $g R g$, so the subrings $e R e+F \cdot 1$ form a directed system. If the induced map

$$
\underset{e \in E}{\operatorname{dir} . \lim } . K_{0}(e R e+F 1) \rightarrow K_{0}(I+F 1)
$$

is a group isomorphism then $K_{0}(I)=G(I)$.

Proof. There is an obvious commutative diagram

$$
\begin{aligned}
& 0 \rightarrow \quad \underset{e \in E}{\operatorname{dir} . \lim } K_{0}(e R e) \rightarrow \quad \underset{e \in E}{\operatorname{dir} . \lim } K_{0}(e R e+F 1) \quad \rightarrow \quad K_{0}(F) \quad \rightarrow \quad 0 \\
& \begin{array}{lllcccc} 
& \rightarrow & \downarrow \alpha & & \downarrow & & \\
K_{0}(I) & \rightarrow & K_{0}(I+F 1) & \rightarrow & K_{0}(F) & \rightarrow & 0
\end{array}
\end{aligned}
$$

with exact rows, and by hypothesis the middle column is an isomorphism so $\alpha$ is also. On the other hand $G$ preserves direct limits, so we have a map $\beta$ : dir.lim. ${ }_{e \in E} G(e R e) \rightarrow G(I)$. As $G(e R e)=K_{0}(e R e)$ for all $e \in E$, it follows that $\beta \alpha^{-1}: K_{0}(I) \rightarrow G(I)$ provides an inverse for $\phi$. Therefore $K_{0}(I)=G(I)$.

We shall need another result. First recall Milnor's definition of the connecting map $\delta: K_{1}(R / I) \rightarrow K_{0}(I, R)$. Consider any element $\mu$ of $K_{1}(R / I)$; it lies in the image of $\mathrm{GL}_{n}(R / I)$ for some $n$ and so can be 
represented as the image of a matrix $u \in M_{n}(R)$ for which there exists $v \in M_{n}(R)$ such that the elements $i=u v-1, j=v u-1$ lie in $M_{n}(I)$. Write

$$
M=\left\{(x, y) \in{ }^{n} R \times{ }^{n} R \mid u(x)-y \in{ }^{n} I\right\} .
$$

In [14, Theorem 2.1] it is proved that $M$ is a finitely generated projective $D(R)$-module. Now $\delta(\mu)$ is defined as $[M]-\left[{ }^{n} D\right]$ and this gives the connecting map. In this situation we have:

\section{Lemma 0.2 As $D$-modules ${ }^{n} D \oplus\left(0 \times i\left({ }^{n} R\right)\right) \approx M \oplus\left(0 \times j\left({ }^{n} R\right)\right)$.}

Proof. By using the Morita equivalence between Mod-D and Mod$M_{n}(D)$ we see that the claimed isomorphism is equivalent to an $M_{n}(D)$ module isomorphism

$$
M_{n}(D) \oplus(0, i) M_{n}(D) \approx{ }^{n} M \oplus(0, j) M_{n}(D) .
$$

It is clear that

$$
{ }^{n} M \approx\left\{(x, y) \in M_{n}(D) \times M_{n}(D) \mid u x-y \in M_{n}(I)\right\}
$$

This shows that without loss of generality we may assume that $n=1$. Now any element of $M$ can be expressed in the form

$$
(x, y)=(1, u)(x, v y)-(0, i)(y, y)
$$

so $M=(1, u) D+(0, i) D$. Now define a $D$-module homomorphism

$$
\alpha: D \oplus(0, i) D \rightarrow M, \quad((x, y),(0, i) d) \mapsto(1, u)(x, y)-(0, i) d .
$$

Clearly $\alpha$ is onto, and $\operatorname{Ker} \alpha=\left\{\left((0, y),\left(0, i y^{\prime}\right)\right) \in D \oplus(0, i) D \mid u y-i y^{\prime}\right.$ $=0\}$. But if $u y-i y^{\prime}=0$ then from the relation

$$
\left(\begin{array}{cc}
-j & v \\
-u & 1
\end{array}\right)\left(\begin{array}{cc}
1 & -v \\
u & -i
\end{array}\right)=\left(\begin{array}{ll}
1 & 0 \\
0 & 1
\end{array}\right)
$$

we obtain

$$
\left(\begin{array}{l}
j \\
u
\end{array}\right)\left(v y^{\prime}-y\right)=\left(\begin{array}{c}
y \\
y^{\prime}
\end{array}\right)
$$

So $\operatorname{Ker} \alpha=((0, j),(0, i u)) D \approx(0, j) D$. Since $M$ is $D$-projective, $\alpha$ splits and the result follows.

1. Regular rings. Let $R$ be a ring.

Recall that $R$ is said to be regular if for every $x \in R$ there exists $y \in R$ such that $x=x y x$. An element $x$ of $R$ is called unit-regular in $R$ if there exists a unit $u$ of $R$ such that $x=x u x$. We say that $R$ is unit-regular if every element in $R$ is unit-regular. 
An ideal $I$ of $R$ has stable range 1 if for all $a, b \in I$, if $(1+a) R+$ $b R=R$ then there exists $c \in I$ such that $(1+a+b c) R=R$; cf. [17], [18]. Vasershtein [17] proves that $I$ having stable range 1 depends only on the rng structure of $I$, and not on the ambient ring $R$. Now one can see that for a ring $R$ the stable range 1 condition is equivalent to saying that for all $a, b \in R, a R+b R=R$ implies $a+b c$ is a unit for some $c \in R$, cf. [18, Theorem 2.6], [2, p. 231].

A theorem of Fuchs and Kaplansky [7, Proposition 4.12] asserts that the unit-regular rings are precisely those regular rings with stable range 1. We shall use Evans' theorem [7, Proposition 4.13]: if the endomorphism ring of a right $R$-module $M$ has stable range 1 , then $M$ can be cancelled from direct sums of right $R$-modules, that is, $M \oplus N \approx M \oplus N^{\prime}$ for some right $R$-modules $N$ and $N^{\prime}$ implies $N \approx N^{\prime}$. By [18, Theorem 2.4, Theorem 3.9] the stable range condition carry over to corners and it is Morita-invariant. Hence, if $R$ has stable range 1 then all finitely-generated projective right $R$-modules cancel from direct sums.

Now we shall give a description for $K_{0}(I)$ in the case where $I$ is an ideal of a regular ring $R$. In an earlier version of this paper we had obtained such a description in the case where $R$ is unit-regular, and then Goodearl provided us with the general case.

First we need a more-or-less known lemma.

LEMMA 1.1. Let $R$ be a regular ring and let $e, f \in R$ be idempotents. Then

(i) If $e R \subseteq f R$, then there exists an idempotent $g$ in $R$ with $g R=f R$ and $g e=e g=e$.

(ii) Let $I$ be an ideal of $R$. If $e, f \in I$ then there exist idempotents $g$, $h \in I$ such that $e R f \subseteq h R h$ and $e R e+f R f \subseteq g R g$. Moreover if $a \in I$ then there exists an idempotent $k \in I$ such that $a \in k R k$.

Proof. (i) Define $g=(1+e f(1-e) f(1-e f(1-e))$.

(ii) Let $h$ be an idempotent such that $e R+f R=h R$. Clearly $h \in I$ and, by (i), we can choose $h$ such that $f h=h f=f$. Then $e R f \subseteq h R h$.

Let $c$ and $d$ be idempotents in $I$ such that $e R+f R=c R$ and $R e+R f=R d$. Then $e R e+f R f \subseteq(e R+f R) \cap(R e+R f)=c R d$. It follows from the above that $c R d \subseteq g R g$, for some idempotent $g$ in $I$.

If $a \in I$, then by regularity there exists $x \in R$ such that $a=a x a$, so $e=a x$ and $f=x a$ are idempotents in $I$ and $a \in e R f$. Now the result follows from the above.

Proposition 1.2 (Goodearl). If $I$ is an ideal of a regular ring $R$ then $G(I)=K_{0}(I)$. 
Proof. Let $E$ be the set of all idempotents in $I$. By Lemma 1.1 (ii), $I+\mathbf{Z} \cdot 1$ is the directed union of the subrings $e R e+\mathbf{Z} \cdot 1$. Therefore dir. $\lim _{e \in E} K_{0}(e R e+\mathbf{Z} \cdot 1)=K_{0}(I+Z \cdot 1)$. By Proposition 0.1 the result follows.

Now we shall obtain a tidier expression for the connecting map $\delta: K_{1}(R / I) \rightarrow K_{0}(I)$ in the case where $R$ is a regular ring.

If $a$ is an $n \times n$ matrix over $R$ we write $\operatorname{Ker} a$ for the set of elements $x \in{ }^{n} R$ such that $a(x)=0$. We define Coker $a$ to be any complement of $a\left({ }^{n} R\right)$ in ${ }^{n} R$, so Coker $a$ is determined up to isomorphism.

Proposition 1.3 (with Goodearl). Let $R$ be a regular ring and $I$ an ideal of $R$. Then the connecting map

$$
\delta: K_{1}(R / I) \rightarrow K_{0}(I)
$$

satisfies $\delta(\bar{a})=[\operatorname{Coker} a]-[\operatorname{Ker} a]$, where $a$ is any matrix over $R$ representing $\bar{a} \in K_{1}(R / I)$.

Proof. Suppose $a \in M_{n}(R)$. By regularity there exists an $n \times n$ matrix $b$ over $R$ such that $a=a b a$. Since $a$ is a unit modulo $I$, we have

$$
\begin{aligned}
& a b-1=i \in M_{n}(I) \\
& b a-1=j \in M_{n}(I) .
\end{aligned}
$$

Now $j\left({ }^{n} R\right)=\operatorname{Ker} a$ and $i\left({ }^{n} R\right) \oplus a\left({ }^{n} R\right)={ }^{n} R$. With the same notation as in Lemma 0.2 we have $\delta(\bar{a})=[M]-\left[{ }^{n} D\right]=[0 \times \operatorname{Coker} a]-[0 \times \operatorname{Ker} a] \in$ $K_{0}(I, R)$. Hence $\delta(\bar{a})=[$ Coker $a]-[\operatorname{Ker} a] \in K_{0}(I)$.

We now use the preceding propositions to obtain some results on lifting units.

LEMMA 1.4. If $R$ is a regular ring and $I$ is an ideal of $R$ then the following are equivalent

(i) For each idempotent $e$ in I the corner ring eRe is unit-regular.

(ii) $I+Z$ is a unit-regular subring of $R$, where $Z$ is the centre of $R$.

(iii) I has stable range 1.

Proof. (i) $\Rightarrow$ (ii) By Lemma 1.1, $I+Z$ is the directed union of the subrings $e R e+Z$, where $e$ is an idempotent in $I$. Now $e R e+Z \approx e R e \times$ $(1-e) Z$ is the direct product of two unit-regular rings, so $e R e+Z$ is unit-regular. Since unit regularity is preserved by taking direct limits we see that $I+Z$ is unit-regular. 
(ii) $\Rightarrow$ (iii) By hypothesis $I+Z$ is unit-regular and so has stable range 1. It follows from [18, Theorem $3.6(\mathrm{~g})]$ that $I$ has stable range 1.

(iii) $\Rightarrow$ (i) Every corner of a rng with stable range 1 also has stable range $1 \mathrm{cf}$. [18, Theorem 3.9].

It follows from [17, Theorem 4] that the sum of two ideals with stable range 1 has stable range 1 . Hence there is a unique largest ideal $R_{0}$ of $R$ having stable range 1 , namely, the sum of all ideals of $R$ with stable range 1.

If an $R$-module $A$ is isomorphic to a direct summand of an $R$-module $B$ then we write $A \lesssim B$. Two idempotents $e$ and $f$ of $R$ are said to be isomorphic if the modules $A=e R, B=f R$ are isomorphic. The notations $e \leq f$ and $e \leq f$ mean $e R \subseteq f R$ and $e R \leq f R$ respectively.

LEMMA 1.5. If $R$ is a regular ring then $R_{0}$ coincides with the ideal $I$ generated by all idempotents of $R$ whose corner is unit-regular.

Proof. By Lemma 1.1 any ideal of $R$ is the directed union of its corners, so by Lemma 1.4 (i) $\Leftrightarrow$ (iii), we see that $R_{0} \subseteq I$.

Conversely, if $e$ is an idempotent in $I$ then $e=\sum x_{i} e_{i} y_{i}$, where $x_{i}$, $y_{i} \in R$ and the $e_{i}$ 's are idempotents with $e_{i} R e_{i}$ unit-regular. From the $R$-linear map $\oplus e_{i} R \rightarrow R, \sum e_{i} r_{i} \mapsto \sum x_{i} e_{i} r_{i}$ we see that $e R \lessgtr \oplus e_{i} R$. It follows from [12, Corollary 10(ii)] that the endomorphism ring of the $R$-module $\oplus e_{i} R$ has stable range 1 and since $e R e$ is a corner of this endomorphism ring it also has stable range 1 .

If $I$ is an ideal of $R$ write $\bar{x}$ for $x+I \in R / I$ and denote by $\pi$ the natural projection $R \rightarrow R / I$.

Proposition 1.6. Let $R$ be a regular ring and $I$ an ideal of $R$ with stable range 1 , then the map

$$
\alpha: U(R / I) \rightarrow K_{0}(I), \quad \bar{a} \mapsto[\operatorname{Coker} a]-[\operatorname{Ker} a],
$$

is a group homomorphism. Moreover

$$
\operatorname{Ker} \alpha=\pi(U(R))=\{\bar{a} \in U(R / I): \text { a is unit-regular }\} .
$$

Proof. By Proposition 1.3 we see that $\alpha$ is the composition of the maps $U(R / I) \rightarrow K_{1}(R / I)$ and $\delta: K_{1}(R / I) \rightarrow K_{0}(I)$ and so it is a group homomorphism.

If $Z$ is the centre of $R$ then $K_{0}(I)$ is a subgroup of $K_{0}(I+Z)$. Notice that $\bar{a}$ lies in $\operatorname{Ker} \alpha$ if and only if [Coker $a]=[\operatorname{Ker} a]$ in $K_{0}(I+Z)$. 
Since $I$ and so $I+Z$ has stable range 1 , we have that $\bar{a} \in \operatorname{Ker} \alpha$ if and only if Coker $a \approx \operatorname{Ker} a$ and this occurs if and only if $a$ is unit-regular, cf. [7, Proof of Theorem 4.1].

Conversely, let $\bar{a} \in \operatorname{Ker} \alpha$, If $a$ is a representative in $R$ for $\bar{a}$, then $a=a u a$ for some unit $u$ in $R$. Now since $\bar{a} \in U(R / I),\left(\bar{a}-\bar{u}^{-1}\right) \bar{u}=0$ so $\bar{a}=\bar{u}^{-1}$ and $\bar{a}$ belongs to $\left.\pi(U / R)\right)$.

Now we consider regular right self-injective rings. The reader is referred to [7] for background. We mention, however, that every regular right self-injective ring can be uniquely expressed as a direct product of a unit-regular ring and a purely infinite regular ring (recall that an idempotent $e$ of a ring $R$ is said to be purely infinite if $(e R) \approx(e R)^{2}$, so $R$ is a purely infinite regular right self-injective ring if 1 is a purely infinite idempotent in $R$ ).

LEMMA 1.7. If $R$ is a purely infinite regular right self-injective ring and I is an ideal of $R$, then $\pi(U(R))=U(R / I)^{\prime}$.

Proof. By [13, Corollary 2.8] $U(R)$ is a perfect group. Hence $\pi(U(R)) \subseteq U(R / I)^{\prime}$.

Conversely, take $u$ in the commutator group $U(R / I)^{\prime}$. Since $R \approx R^{2}$ there exist matrices $X \in R^{2}$ and $Y \in{ }^{2} R$ such that $X Y=1$ and $Y X=I_{2}$. Then $\bar{Y} u \bar{X}$ is a $2 \times 2$ invertible matrix. By [13, Theorem 2.2] $\bar{Y} u \bar{X} \in$ $E_{2}(R / I)$, hence there exists $Z \in \mathrm{GL}_{2}(R)$ such that $\bar{Z}=\bar{Y} u \bar{X}$. Therefore $v=X Z Y$ is a unit of $R$ with $\bar{v}=u$. The result follows.

If $e$ is an idempotent of a regular right self-injective ring, then we denote by $\operatorname{cc}(e)$ its central cover, that is, the minimum central idempotent such that $\operatorname{cc}(e) e=e$.

Proposition 1.8. Let $R$ be a purely infinite regular right self-injective ring and $I$ an ideal. If $A, B \in P(I)$, then

(i) $[A]=[B] \in K_{0}(I)$ if and only if there exists a purely infinite idempotent $e$ in I such that $A \oplus e R \approx B \oplus e R$.

(ii) (with Goodearl) $K_{0}(I)=0$ if and only if every idempotent in $I$ is sub-isomorphic to a purely infinite idempotent in $I$.

(iii) $[A]=[B] \in K_{0}(I)$ if and only there exists a purely infinite idempotent $e$ in I such that $A \oplus \operatorname{cc}(e) R \approx B \oplus \operatorname{cc}(e) R$.

(iv) $[A]=[B] \in K_{0}(I)$ if and only if there exists a purely infinite idempotent $e$ in I such that $(1-\operatorname{cc}(e)) A \approx(1-\operatorname{cc}(e)) B$. 
Proof. (i) By Proposition 1.2, $K_{0}(I)=G(I)$. Thus $[A]=[B]$ if and only if $A \oplus C \approx B \oplus C$ for some $C \in P(I)$. It follows from [7, Theorem 10.32] that $C$ can be written as $C_{1} \oplus C_{2}$, where $C_{2}$ is purely infinite and the endomorphism ring of $C_{1}$ has stable range 1 . But then $C_{1}$ cancels from direct sums and we have $A \oplus C_{2} \approx B \oplus C_{2}$. Since $R \approx R^{2}, C_{2}$ is cyclic and so $C_{2} \approx e R$, for some purely infinite idempotent $e$ in $I$.

(ii) Since $R$ is purely infinite, we see that every finitely generated right $R$-module is cyclic.

Suppose $K_{0}(I)=0$ and let $e$ be an idempotent in $I$. By (i) there exists a purely infinite idempotent $f$ in $I$ such that $e R \oplus f R \approx f R$. Thus $e R \lesssim f R$ as desired.

Conversely, let $e$ be an idempotent in $I$. By hypothesis $e R \leqslant f R$ for some purely infinite idempotent $f$ in $I$. Then, since $f R \leqslant e R \oplus f R \lessgtr$ $(f R)^{2}$, by [7, Theorem 10.14] we have $e R \oplus f R \approx f R$. So $[e R]=0$ in $K_{0}(I)$.

(iii) Suppose $[A]=[B] \in K_{0}(I)$. By (i), $A \oplus e R \approx B \oplus e R$ for some purely infinite idempotent $e$ in $I$ and a fortiori $A \oplus \operatorname{cc}(e) R \approx B \oplus$ $\operatorname{cc}(e) R$.

Conversely, if $A \oplus \operatorname{cc}(e) R \approx B \oplus \operatorname{cc}(e) R$ then we have $(1-\operatorname{cc}(e)) A$ $\approx(1-\operatorname{cc}(e)) B$. Hence it suffices to prove that $[\operatorname{cc}(e) A]=[\operatorname{cc}(e) B]=[0]$. By cutting down to $\operatorname{cc}(e) R$ we may assume $e$ faithful and we need only verify $[A]=[0]$. Thus we are reduced to the case $A$ directly finite. By the general comparability axiom there exists a central idempotent $h$ such that $h e R \leqslant h A$ and $(1-h) A \lessgtr(1-h) e R$. Since $h A$ is directly finite and $h e R$ purely infinite we deduce that $h e R=0$. But $e$ is faithful so $h=0$. Then $A \lesssim e R$ and the result follows from the proof of (ii).

(iv) The relation $A \oplus \operatorname{cc}(e) R \approx B \oplus \operatorname{cc}(e) R$ is equivalent to $(1-\operatorname{cc}(e)) A \approx(1-\operatorname{cc}(e)) B$ and $\operatorname{cc}(e) A \oplus \operatorname{cc}(e) R \approx \operatorname{cc}(e) B \oplus \operatorname{cc}(e) R$. Since $\operatorname{cc}(e)$ is purely infinite the latter relation always holds. So the result follows from (iii).

THEOREM 1.9. Let $R$ be a purely infinite, regular, right self-injective ring and let $I$ be an ideal of $R$. Then

(i) The map

$$
\alpha: U(R / I) \rightarrow K_{0}(I), \quad \alpha(\bar{a})=[\text { Coker } a]-[\operatorname{Ker} a]
$$

is a group homomorphism which induces an isomorphism

$$
K_{1}(R / I)=U(R / I)^{\mathrm{ab}} \stackrel{\approx}{\rightarrow} K_{0}(I) .
$$

(ii) $A$ unit $\bar{a} \in U(R / I)$ can be lifted to a unit in $R$ if and only if $[$ Coker $a]=[\operatorname{Ker} a] \in K_{0}(I)$. 
Proof. (i) Let $f: U(R / I) \rightarrow K_{1}(R / I)$ be the natural map. It follows from [13, Theorem 1.2 (iii) and Theorem 2.2] that $f$ is onto and $\operatorname{Ker} f=$ $U(R / I)^{\prime}$. So $K_{1}(R / I)=U(R / I)^{\mathrm{ab}}$. By [13, Theorem 2.7 (ii)] $K_{1}(R)=0$ and it follows from [7, Proposition 15.6] that $K_{0}(R)=0$. Thus (i) follows from Proposition 1.3.

(ii) This is an immediate consequence of (i) and Lemma 1.7.

LEMMA 1.10. Let $R$ be a regular right self-injective ring and $I$ an ideal of $R$. If $e$ is an idempotent of $I$, then the following are equivalent

(i) $e \leqslant f$ for some purely infinite idempotent $f$ in $I$.

(ii) $e \leq f$ for some purely infinite idempotent $f$ in $I$.

Proof. Clearly (ii) $\Rightarrow$ (i). Conversely, by [7, Theorem 10.32] there exists a central idempotent $h$ in $R$ such that $h e R$ is purely infinite and $(1-h) e R$ is directly finite. So without loss of generality we may assume that $e R$ is directly finite. We have $e R \approx e^{\prime} R \subseteq f R$ for some idempotent $e^{\prime}$. Since $e R e$ has stable range $1,(1-e) R \approx\left(1-e^{\prime}\right) R$, so there exists a unit $u$ in $R$ such that $e=u^{-1} e^{\prime} u$. The idempotent $u^{-1} f u$ is a purely infinite idempotent in $I$ and $e \leq u^{-1} f u$.

COROLlARY 1.11. Let $R$ be a regular right self-injective ring. Let $e_{1}$ be the central idempotent in $R$ such that $e_{1} R$ is purely infinite and $\left(1-e_{1}\right) R$ is directly finite. Then the following are equivalent

(i) Every unit in $R / I$ can be lifted to a unit in $R$.

(ii) For every idempotent $e \in e_{1} I$ there exists a purely infinite idempotent $f \in I$ such that $e \leq f$.

(iii) $K_{0}\left(e_{1} I\right)=0$.

Proof. $R$ decomposes into the direct product of the rings $R_{1}=e_{1} R$ and $R_{2}=\left(1-e_{1}\right) R$. Since $R_{2}$ is unit-regular it is clear that a unit in a factor ring of $R_{2}$ can be lifted to a unit in $R_{2}$. Thus without loss of generality we may assume that $R$ is purely infinite, that is, $e_{1}=1$.

The equivalence (ii) $\Leftrightarrow$ (iii) follows from Proposition 1.8 (ii) and Corollary 1.10. It is clear from Theorem 1.9 (ii) that (i) $\Leftrightarrow$ (iii).

COROLLARY 1.12. If $R$ is a regular right self-injective ring of Type III and $I$ is an ideal of $R$, then every unit in $R / I$ can be lifted to a unit in $R$.

Proof. Since $R$ is Type III every idempotent is purely infinite. The result follows from Corollary 1.11 . 
Now it is a simple matter to extend Corollary 1.11 to arbitrary right self-injective rings. For this we first need a lemma.

For any ring $R$ denote by $J=J(R)$ its Jacobson radical. We shall use the fact that an element of $R$ is a unit if and only if so is modulo $J$. Recall that if $R$ is right self-injective then $R / J$ is regular and right self-injective. Moreover every idempotent in $R / J$ can be lifted to an idempotent in $R$.

We denote by $R_{\infty}$ the right ideal generated by all purely infinite idempotents in $R$.

LEMMA 1.13. If $R$ is right self-injective, then $R_{\infty}$ is an ideal of $R$.

Proof. If $e$ is a purely infinite idempotent in $R$ then it suffices to prove that $x e \in R_{\infty}$ for all $x$ in $R$. In the case $x$ is a unit we have that $x e x^{-1}$ is a purely infinite idempotent, hence $x^{-1} \in R_{\infty}$ and so $x e \in R_{\infty}$. Now write $R / J=R_{1} \times R_{2}$ where $R_{1}$ is purely infinite and $R_{2}$ is unitregular. Let $S_{1}$ and $S_{2}$ be the ideals of $R$ such that $S_{1} / J=R_{1}$ and $S_{2} / J=R_{2}$. Since $R=S_{1} S_{2}$ it suffices to consider separately the cases $x \in S_{1}$ and $x \in S_{2}$.

Suppose first $x \in S_{1}$. Since $R_{1}$ is purely infinite $R_{1} \approx M_{2}\left(R_{1}\right)$ and hence every element of $R_{1}$ is a sum of an even number of units in $R_{1}$. But then, every element of $R_{1}$ is a sum of units in $R_{1} \times R_{2}$ and so every element of $S_{1}$ is a sum of units in $R$. Now it is clear that $x e \in R_{\infty}$.

Assume now $x \in S_{2}$. Since $R_{2}$ is unit-regular we can find an idempotent $f$ and $a$ unit $u$ in $R$ such that $x u-f \in J$. So $x-f u^{-1}$ is a sum of two units. On the other hand $f R e \subseteq J$ so also $f u^{-1} e$ is a sum of two units. Therefore $x e=\left(x-f u^{-1}\right) e+f u^{-1} e \in R_{\infty}$.

THEOREM 1.14 If $R$ is a right self-injective ring and $I$ is an ideal of $R$, then the following are equivalent.

(i) Every unit in $R / I$ can be lifted to a unit in $R$.

(ii) If $e$ is an idempotent in $I$ which is contained in a purely infinite idempotent in $R$, then there exists a purely infinite idempotent in I containing $e$.

(iii) $K_{0}\left(I R_{\infty}\right)=0$.

Proof. Write $\bar{R}=R / J$ and denote images in $\bar{R}$ by overbars. Note that $R /(I+J)$ is a factor ring of the regular ring $R / J$. So $J(R /(I+J))$ $=0$. Therefore $J(R / I)=(I+J) / I$. Now we have the following commutative diagram

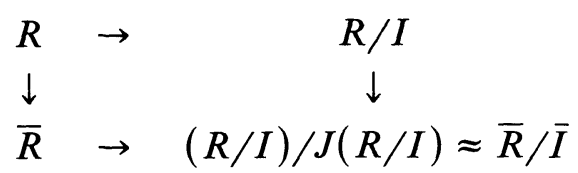


where the rows and columns are the natural projections. Now it is easily seen that $U(R) \rightarrow U(R / I)$ is onto if and only if $U(\bar{R}) \rightarrow U(\bar{R} / \bar{I})$ so is.

If $e_{1} \bar{R}$ is the purely infinite part of $\bar{R}$, then $\overline{I R}_{\infty}=e_{1} \bar{I}$. Thus $K_{0}\left(e_{1} \bar{I}\right) \approx K_{0}\left(I R_{\infty}\right)$ (for this notice that the kernel of the natural projection $I R_{\infty} \rightarrow e_{1} \bar{I}$ is contained in $\left.J\right)$. Now it follows from Corollary 1.11, applied to the pair $(\bar{R}, \bar{I})$ that (i) $\Leftrightarrow$ (iii). The result will follow by using Corollary 1.11 and noting that (ii) holds for the pair $(R, I)$ if and only if it holds for $(\bar{R}, \bar{I})$.

Suppose first that $(\bar{R}, \bar{I})$ satisfies (ii). Let $e$ be an idempotent in $I$ such that $e \leq f$ for some purely infinite idempotent $f$ in $R$. Then $\bar{e} \leq \bar{f}$ and so there exists a purely infinite idempotent $g$ in $R$ such that $\bar{e} \leq \bar{g}$ and $\bar{g}$ belonging to $\bar{I}$. In fact $g \in I+J$ and thus $g \in I$.

Now we have $\bar{g} \bar{e}=\bar{e}$ so $g e-e=j \in J$. From this we easily obtain $g(1+j) e=(1+j) e$. But then $g_{1}=(1+j)^{-1} g(1+j)$ is a purely infinite idempotent in $I$ such that $e=g_{1} e \leq g_{1}$.

Conversely, let $\bar{e}$ be an idempotent in $\bar{I}$ such that $\bar{e} \leq \bar{f}$ for some purely infinite idempotent $\bar{f}$ in $\bar{R}$. Clearly we may assume $f$ is a purely infinite idempotent in $R$ and $e$ is an idempotent in $I$. Then $f e-e=j \in J$. As in the preceding paragraph we obtain $e \leq f_{1}=(1+j)^{-1} f(1+j)$. Clearly $f_{1}$ is purely infinite and so, by hypothesis, there exists a purely infinite idempotent $g$ in $I$ with $e \leq g$. Therefore $g \in I$ is a purely infinite idempotent such that $\bar{e} \leq \bar{g}$.

COROLlaRY 1.15. If $R$ is a prime, regular, right self-injective ring, and $I$ is an ideal of $R$, then

(i) If $I=R_{0}$, then a unit $\bar{a} \in R / I$ can be lifted to a unit in $R$ if and only if $a$ is unit regular or equivalently $\operatorname{Ker} a \approx \operatorname{Coker} a$.

(ii) If $I \neq R_{0}$, then every unit in $R / I$ can be lifted to a unit in $R$.

Proof. (i) It follows from Proposition 1.6.

(ii) If $I \neq R_{0}$ then, by Lemma 1.5, there exists an idempotent $e$ in $I$ such that $e R e$ is not unit-regular, but $R$ being prime, regular, right self-injective this implies that $e$ is purely infinite. By Theorem 1.14 we must prove that every idempotent $f$ in $I$ is contained in a purely infinite idempotent in $I$. Without loss of generality we way assume that $f$ is directly finite. Since $R$ satisfies the comparability axiom we have either $e \lesssim f$ or $f \leqq e$. Since $e \neq 0$ we must have $f \lesssim e$, as desired.

EXAMPLE. Let $R=\operatorname{End}_{k}(V)$ where $V$ is an infinite-dimensional $K$-vector space. In this case $R_{0}=\left\{x \in R \mid \operatorname{dim}_{K} x(V)<\infty\right\}$. If we associate with each $[e R] \in K_{0}\left(R_{0}\right)$ the $K$-dimension of $e(V)$, we obtain an 
isomorphism $K_{0}\left(R_{0}\right) \stackrel{\approx}{\rightarrow} \mathbf{Z}$. By Theorem $1.9 U\left(R / R_{0}\right)^{\text {ab }} \approx \mathbf{Z}$, furthermore a unit $\bar{a}$ in $R / R_{0}$ can be lifted to a unit in $R$ if and only if $\operatorname{dim}_{K}$ Coker $a=\operatorname{dim}_{K} \operatorname{Ker} a$.

2. Computation of $K_{0}(I)$. Let $R$ be a purely infinite regular right self-injective ring and let $I$ be an ideal of $R$. Our goal now is to realize $K_{0}(I)$ as a group of continuous functions. This has been motivated by Olsen's work in $W^{*}$-algebras [15].

The starting point in Olsen's proof is Wils' characterization of the closed ideals of $W^{*}$-algebras. Although in the regular case such a characterization is not our disposal, we can obtain our results by extending some computations due to Goodearl and Boyle.

If $M$ is a right $R$-module and $n \geq 0$ is an integer we shall write $n M$ for $M^{n}$.

LEMMA 2.1 Let $R$ be a regular ring. Let $A$ and $B$ be nonsingular injective right $R$-modules such that the endomorphism ring $\operatorname{End}_{R} A$ is Type II and $p A \approx q B$ for some positive integers $p, q$. Let $r$ be a positive integer.

(i) If $r \leq p$ then there exists a right $R$-module $D$ such that $D \subseteq B$ and $q D \approx r A$.

(ii) Assume $A$ is directly finite. Let $C$ be a finitely generated projective right $R$-module such that $A, B \subseteq C$ and $r A \leq q C$. If $r \geq p$ then there exists $a$ right $R$-module $D$ such that $B \subseteq D \subseteq C$ and $q D \approx r A$.

Proof. (i) We have $r A \approx B_{1} \subseteq q B$ for some $B_{1}$. Since End $B_{R} B_{1}$ is Type II (see [7, Theorem 10.10]) by [7, Proposition 10.28] $B_{1} \approx q B_{2}$ for some $B_{2}$. So $q B_{2} \leq q B$ and by [7, Theorem 10.34] there exists a right $R$-module $D$ such that $B_{2} \approx D \subseteq B$.

(ii) As in (i) there exists $A_{1} \subseteq C$ such that $r A \approx q A_{1}$. Now consider the submodule of $C, B+A_{1}$, which is finitely generated and so projective. Then $B+A_{1} \leq B \oplus A_{1}$ and by [7, Corollary 9.20] $B+A_{1}$ is a directly finite nonsingular injective right $R$-module. Thus $\operatorname{End}_{R}\left(B+A_{1}\right)$ is unit regular.

On the other hand $q B \approx p A \subseteq r A \approx q A_{1}$, so $B \approx B_{1} \subseteq A_{1}$ for some $B_{1}$. Then by [7, Corollary 4.4] there are decompositions $B+A_{1}=B \oplus B^{\prime}$ $=B_{1} \oplus B^{\prime}$ and thus $D=B \oplus\left(A_{1} \cap B^{\prime}\right)$ is the desired $R$-module.

Finally note that (i) follows for any ring $R$.

LEMMA 2.2. Let $R$ be a regular right self-injective ring. Let $A$ be a principal right ideal of $R$ such that $\operatorname{End}_{R} A$ is Type $\mathrm{II}_{f}$. Let $\left\{p_{n}, q_{n}\right\}_{n \in \mathbf{N}}$ be $a$ set of positive integers such that $p_{n} A \leqq q_{n} R$ for every $n$. Then there exist 
principal right ideals of $R ; B_{1}, B_{2}, \ldots$ such that $q_{n} B_{n} \approx p_{n} A$ for every $n$ and $B_{n} \subseteq B_{m}$ whenever $p_{n} / q_{n} \leq p_{m} / q_{m}$.

Proof. We are going to construct the right ideals $B_{n}$ by induction on $n$. Since $p_{1} A \leq q_{1} R$ and $\operatorname{End}_{R} A$ is Type II there exists a principal right ideal $A_{1}$ such that $p_{1} A \approx p_{1} q_{1} A_{1} \leqslant q_{1} R$ cf. [7, Proposition 10.28]. Then by [7, Theorem 10.34] $p_{1} A_{1} \approx B_{1} \subseteq R$ for some right ideal $B_{1}$.

Now suppose we have constructed $B_{1}, \ldots, B_{n}$. Set $\lambda_{n}=p_{n} / q_{n}$ for each $n$. Assume for simplicity that $\lambda_{1} \geq \lambda_{2} \geq \cdots \geq \lambda_{n}$. Now there are three possibilities: (1) $\lambda_{n+1} \leq \lambda_{n}$, (2) $\lambda_{1} \leq \lambda_{n+1}$ and (3) $\lambda_{i} \geq \lambda_{n+1} \geq \lambda_{i+1}$ for some $i \in\{1, \ldots, n-1\}$.

(1) By the induction hypothesis we have $q_{n} B_{n} \approx p_{n} A$, so $q_{n+1} q_{n} B_{n} \approx$ $q_{n+1} p_{n} A$ and then, by applying Lemma 2.1(i), there exists a principal right ideal $B_{n+1}$ with $B_{n+1} \subseteq B_{n}$ and $q_{n+1} B_{n+1} \approx p_{n+1} A$.

(2) Let $A_{1}$ be a submodule of $A$ such that $A \approx q_{n+1} A_{1}$. Now $q_{1} B_{1} \approx p_{1} A \approx p_{1} q_{n+1} A_{1}$. On the other hand $p_{n+1} q_{n+1} A_{1} \lesssim q_{n+1} R$ implies $p_{n+1} A_{1} \lesssim R$. By Lemma 2.1 (ii) there exists $B_{n+1}$ with $B_{1} \subset B_{n+1}$ and $q_{1} B_{n+1} \approx p_{n+1} q_{1} A_{1}$, thus $q_{n+1} B_{n+1} \approx p_{n+1} A$.

(3) As in the case (1), there exists a submodule of $B_{i}$, say $B$, such that $q_{n+1} B \approx p_{n+1} A$. From the relation $\lambda_{n+1} \geq \lambda_{i+1}$ we obtain $p_{i+1} q_{n+1} B_{i+1}$ $\lesssim p_{n+1} q_{i+1} B_{i+1} \approx p_{i+1} p_{n+1} A \approx p_{i+1} q_{n+1} B$, so there exists $B_{i+1}^{*}$ with $B_{i+1}$ $\approx B_{i+1}^{*} \subseteq B$. Then by [7, Corollary 4.4] there are decompositions $B+$ $B_{i+1}=B_{i+1} \oplus B^{*}=B_{i+1}^{*} \oplus B^{*}$.

Now write $B_{n+1}$ for the module $B_{i+1} \oplus\left(B \cap B^{*}\right)$. Then $B_{i} \supseteq B_{n+1}$ $\supseteq B_{i+1}$ and $q_{n+1} B_{n+1} \approx p_{n+1} A$.

Let $R$ be a regular right self-injective ring. If $e$ is a directly finite idempotent of $R$, then $e R e$ is unit-regular cf [7; Corollary 1.23, Theorem 9.17]. By Lemma 1.5 we see that $R_{0}$ coincides with the ideal of $R$ generated by all directly finite idempotents of $R$.

LEMMA 2.3. Let $R$ be a regular right self-injective ring and $I$ an ideal of $R$ contained in $R_{0}$. If $J$ is an ideal of $R$ contained in $I$, then the natural homomorphism $K_{0}(J) \rightarrow K_{0}(I)$, induced by the inclusion $J \subseteq I$, is injective.

Proof. By Proposition 1.2 every element in $K_{0}(J)$ can be written in the form $[A]-[B]$ for some finitely generated projective right $R$-modules in $P(I)$. If $[A]=[B]$ in $K_{0}(I)$, then there exists a finitely projective right $R$-module $C \in P(I)$ with $A \oplus C \approx B \oplus C$. Since every idempotent in $I$ 
is directly finite, by [7, Corollary 9.20] $C$ is directly finite and then by [7, Corollary 9.18] $A \approx B$. So $[A]=[B]$ in $K_{0}(J)$.

From now on we shall identity $K_{0}(J)$ with its image in $K_{0}(I)$.

Let $B(R)$ be the set of all central idempotents of $R$. If $\left\{e_{i}\right\}_{i \in I}$ is a family of elements in $R$ we denote by $\bigvee_{i \in I} e_{i}$ and by $\bigwedge_{i \in I} e_{i}$ its supremun and its infimum respectively. If $R$ is regular and right self-injective then by [7, Proposition 9.9] $B(R)$ is a complete Boolean algebra.

Let $X=B S(R)$ be the Boolean spectrum of $R$, that is, $X$ is the set of all maximal ideals of $B(R)$. Recall that the closed sets in $X$ are of the form $V(S)=\{M \in B S(R) \mid S \subseteq M\}$, where $S \subseteq B(R)$. Recall that with this topology, $X$ is an Stonian space, that is, $X$ is a compact Hausdorff space such that the closure of every open set is open. If $Y \subseteq X$ then we denote the closure of $Y$ in $X$ by $\bar{Y}$.

We shall need the following simple lemma.

LEMMA 2.4. Suppose $\left\{e_{i}\right\}_{i \in I}$ is a family of elements in $B(R)$. If $X_{i}=V\left(1-e_{i}\right)$ for all $i$, then $\bar{\bigcup}_{i \in I} X_{i}=V\left(1-\bigvee_{i \in I} e_{i}\right)$.

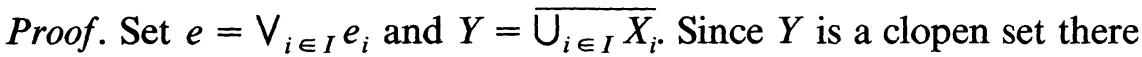
exists $f$ in $B(R)$ such that $Y=V(1-f)$. It is easily seen that the inclusion $X_{i}=V\left(1-e_{i}\right) \subseteq Y=V(1-f)$ implies $e_{i} \leq f$ for all index $i$. So $e \leq f$. On the other hand we have $\cup_{i \in I} X_{i} \subseteq V(1-e)$. Because $V(1-e)$ is clopen it contains $Y$. So $f \leq e$.

Let $f: X \rightarrow[-\infty, \infty]$ be a continuous map of $X$ into the extended real interval $[-\infty, \infty]$. We say that $f$ is almost finite if it is finite in a dense open subset of $X$. We denote by $\mathscr{C}(X,[-\infty, \infty])$ the set of all almost finite continuous maps of $X$ into $[-\infty, \infty]$. Assume $f, g \in$ $\mathscr{C}(X,[-\infty, \infty])$ and let $U$ be a dense open set in $X$ such that $f$ and $g$ are finite in $U$. Consider the continuous map $f+g$ of $U$ into $[-\infty, \infty]$ defined with pointwise addition. Since $X$ is Stonian, $\bar{U}=X$ is the Stone-Čech compactification of $U$ (see [19, 1.14 Theorem]), then, in particular, by [19, 1.11 Theorem] $f+g$ can be extended to a unique continuous map, also denoted by $f+g$, of $X$ into $[-\infty, \infty]$. With this addition and the natural order, $\mathscr{C}(X,[-\infty, \infty])$ becomes an ordered abelian group.

Let $G$ be a partially ordered abelian group and let $H$ be a subgroup of $G$. Recall that $H$ is said to be directed if it is upward directed, and convex if whenever $x_{1}, x_{2} \in H$ and $y \in G$ such that $x_{1} \leq y \leq x_{2}$, then 
$y \in H$. It is known (see for example [7, Proposition 15.17]) that the set of all directed convex subgroups of $G$ ordered by inclusion forms a lattice denoted by $L(G)$.

For any rng $I$ we denote by $L_{2}(I)$ the lattice of ideals of $I$.

For the definition of the relative dimension functions on the nonsingular injective right modules over regular right self-injective rings we refer to [7, Chapter 11].

THEOREM 2.5. Let $R$ be a regular right self-injective ring of Type $\mathrm{II}_{\infty}$ and let $e_{0}$ be a faithful directly finite idempotent in $R$. Then

(i) The rule

$$
[e R] \mapsto \varphi_{e}, \quad \varphi_{e}(M)=d_{M}\left(e R: e_{0} R\right)
$$

defines an isomorphism of partially ordered abelian groups.

$$
\varphi: K_{0}\left(R_{0}\right) \stackrel{\approx}{\rightarrow} \mathscr{C}(X,[-\infty, \infty])
$$

(ii) The map

$$
L_{2}\left(R_{0}\right) \rightarrow L\left(\mathscr{C}(X,[-\infty, \infty]), \quad J \mapsto \varphi\left(K_{0}(J)\right)\right)
$$

is a lattice isomorphism.

Proof. (i) Denote by $\mathscr{C}(X,[0, \infty])$ the set of all almost finite continuous maps of $X$ into the extended real interval [0, $\infty$. By [7, Lemma 11.16] if $e$ is an idempotent in $R_{0}$ then the map

$$
\varphi_{e}: X \rightarrow[0, \infty], \quad M \mapsto d_{M}\left(e R: e_{0} R\right)
$$

is continuous. Now we prove that in fact $\varphi_{e}$ belongs to $\mathscr{C}(X,[0, \infty])$. Set $U=\varphi_{e}^{-1}([0, \infty))$, which is an open set. Because $X$ is Stonian, $\bar{U}$ is clopen and so $\bar{U}=V(f)$ for some $f$ in $B(R)$. Suppose $e g \leqslant n e_{0} g$ for some positive integer $n$ and some central idempotent $g$. If $f g \neq 0$ then there exists a maximal ideal $M$ in $B(R)$ such that $f g \notin M$, thus $d_{M}\left(e R: e_{0} R\right) \leq n$ and so $M \in V(f)$, which is a contradiction. Then $f g=$ 0 . Let $m$ be a positive integer. By the general comparability axiom there exists a central idempotent $h$ such that $e f h \lessgtr m e_{0} f h$ and $(1-h) m e_{0} f \lesssim$ ef $(1-h)$. Then by the above $f h=0$ and so $m e_{0} f \leqslant e f$. Since this holds for all $m$ we see that $e_{0} f=0, \operatorname{cf}[7$, Corollary 9.23]. Therefore $f=0$ and $\bar{U}=X$.

Since $R$ is purely infinite, for every finitely projective right $R$-module $A$ there exists an idempotent $e$ in $R$ such that $A \approx e R$. Thus we have a well-defined map

$$
\varphi: K_{0}\left(R_{0}\right)^{+} \rightarrow \mathscr{C}(X,[0, \infty]), \quad[e R] \mapsto \varphi_{e}
$$

where $e$ is any idempotent of $R_{0}$. 
Now we prove that $\varphi$ is onto. For this let $\alpha$ be an element in $\mathscr{C}(X,[0, \infty])$. Let $X_{0}$ denote the closure of the set $\{M \in X \mid \alpha(M)>0\}$. For any integers $m$ and $n$ such that $m \geq 0$ and $n \geq 1$ let $X_{m n}$ denote the closure of the set $\left\{M \in X \mid \alpha(M)>m / 2^{n}\right\}$. Note that $X_{0 n}=X_{0}$ and $X_{m n} \subseteq X_{m-1, n}$ for all $m$ and $n$. It is easily seen that $X_{0}-\alpha^{-1}(\infty)=$ $\cup_{m=1}^{\infty}\left(X_{m-1, n}-X_{m n}\right)$ for a fixed $n$. Since $\alpha$ is almost finite, $X_{0}-\alpha^{-1}(\infty)$ $=X_{0}$. Suppose $X_{m-1, n}-X_{m n}=\left\{M \in X \mid e_{m n} \notin M\right\}$ for all $m$ and $n$ and for some $e_{m n}$ in $B(R)$. It is clear that for each $n$ the $e_{m n}$ 's are orthogonal because the sets $X_{m-1, n}-X_{m n}$ are disjoint. It follows from Lemma 2.4 that $X_{0}=\{M \in X \mid 1-e \notin M\}$, where $1-e=\bigvee_{m, n} e_{m n}$.

For any $m$ and $n, X_{m-1, n}=X_{2 m-2, n+1}$ and $X_{m-1, n}-X_{m n}$ is the disjoint union of $X_{2 m-2, n+1}-X_{2 m-1, n+1}$ and $X_{2 m-1, n+1}-X_{2 m, n+1}$. So $e_{m n}=e_{2 m-1, n+1}+e_{2 m, n+1}$. Let $f_{m n}$ be an idempotent such that $2^{n} f_{m n} R$ $\approx m e_{0} R$. Since $e_{0} R$ is directly finite it follows from [7, Proposition 11.3(e)] that $d_{M}\left(f_{m n} R: e_{0} R\right)=m / 2^{n}$ for all $M$ in $X$. By Lemma 2.2 we can assume that $f_{m n} \leq f_{s t}$ if $m / 2^{n} \leq s / 2^{t}$.

Let $A_{n}=\mathrm{V}_{m \geq 1}\left(e_{m n} f_{m-1, n} R\right)$ and note that $A_{n}$ is an injective hull of $\oplus_{m \geq 1} e_{m n} f_{m-1, n} R$. It is easily seen that $A_{n}$ is directly finite. Now we have

$$
\begin{aligned}
\bigoplus_{m \geq 1} e_{m n} f_{m-1, n} R=\bigoplus_{m \geq 1}\left(e_{2 m-1, n+1}+e_{2 m, n+1}\right) f_{m-1, n} R \\
\subseteq\left(\bigoplus_{m \geq 1} f_{2 m-2, n+1} e_{2 m-1, n+1} R\right) \oplus\left(\bigoplus_{m \geq 1} f_{2 m-1, n+1} e_{2 m, n+1} R\right) \\
\quad=\bigoplus_{m \geq 1} f_{m-1, n+1} e_{m, n+1} R .
\end{aligned}
$$

So $A_{n} \subseteq A_{n+1}$ for all $n$. Set $A=\bigcup_{n \geq 1} A_{n}$.

For any integer $t \geq 1$ define $A_{t}^{*}=\left(\bigvee_{m \geq 1} f_{m t} e_{m t} R\right)$. As above $A_{t}^{*}$ is directly finite and we have

$$
\begin{aligned}
\bigoplus_{m \geq 1} f_{m, t+1} e_{m, t+1} R & =\left(\bigoplus_{j \geq 1} f_{2 j-1, t+1} e_{2 j-1, t+1} R\right) \oplus\left(\bigoplus_{j \geq 1} f_{2 j, t+1} e_{2 j, t+1} R\right) \\
& \subseteq \bigoplus_{j \geq 1} f_{2 j, t+1}\left(e_{2 j-1, t+1}+e_{2 j, t+1}\right) R \\
& =\bigoplus_{j \geq 1} f_{2 j, t+1} e_{j, t} R=\bigoplus_{j \geq 1} f_{j t} e_{j t} R .
\end{aligned}
$$

So $A_{t+1}^{*} \subseteq A_{t}^{*}$. Now $A_{t} \subseteq A_{t}^{*}$ and then $A \subseteq A_{t}^{*}$.

We shall prove that $\varphi([A])=\alpha$. Since $\alpha$ is almost finite we must show that $\varphi([A])(M)=\alpha(M)$ for all $M \in X-\alpha^{-1}(\infty)$. If $e \notin M$ then $d_{M}\left(A: e_{0} R\right)=d_{M}\left(A e: e_{0} R\right)=0$ because $A e=0$. Now suppose that $e$ 
belongs to $M$. Then for each $n$ we have that there exists an $m$ such that $M \in X_{m-1, n}-X_{m, n}$. So $m-1 / 2^{n} \leq \alpha(M) \leq m / 2^{n}$. Since $A_{n} e_{m n} R=$ $f_{m-1, n} e_{m n} R$ then $d_{M}\left(A_{n}: e_{0} R\right)=m-1 / 2^{n}$ and so $d_{M}\left(A: e_{0} R\right) \geq$ $(m-1) / 2^{n}$. Similarly $d_{M}\left(A: e_{0} R\right) \leq d_{M}\left(A_{n}^{*}: e_{0} R\right)=d_{M}\left(A_{n}^{*} e_{m n}: e_{0} R\right)=$ $d_{M}\left(f_{m n} R: e_{0} R\right)=m / 2^{n}$. Then $\varphi([A])(M)-1 / 2^{n} \leq \alpha(M) \leq \varphi([A])(M)$ $+1 / 2^{n}$ for all $n$. So $\alpha(M)=\varphi([A])(M)$.

Now by [7, Theorem 11.11] the map

$$
\varphi: K_{0}\left(R_{0}\right) \rightarrow \mathscr{C}(X,[-\infty, \infty]), \quad[e R]-[f R] \rightarrow \varphi_{e}-\varphi_{f}
$$

is a group homomorphism. By [7, Theorem 11.15 (a)] $\varphi$ is an order preserving homomorphism. Because any element of $\mathscr{C}(X,[-\infty, \infty])$ can be written as a difference of two elements of $\mathscr{C}(X,[0, \infty])$, by the preceding paragraph it is clear that $\varphi$ is onto. To prove injectivity suppose $\varphi_{e}=\varphi_{f}$ for some idempotents $e$ and $f$ in $K_{0}\left(R_{0}\right)$. Then by [7, Theorem $11.15(\mathrm{~b})] e R \approx f R$ and so $[e R]=[f R]$ in $K_{0}\left(R_{0}\right)$.

(ii) If $J$ is an ideal of $R$ contained in $R_{0}$, by Lemma $2.3 K_{0}(J)$ is a subgroup of $K_{0}\left(R_{0}\right)$. Now, as in the proof of [7, Theorem 15.20] one can see that the correspondence $J \mapsto K_{0}(J)$ defines a lattice isomorphism of $L\left(R_{0}\right)$ onto $L\left(K_{0}\left(R_{0}\right)\right)$. Since $\varphi$ is an order group isomorphism, the result follows.

COROLlARY 2.6. If $R$ is a prime regular right self-injective ring of Type $\mathrm{II}_{\infty}$, then $K_{1}\left(R / R_{0}\right)=U\left(R / R_{0}\right)^{\mathrm{ab}} \approx K_{0}\left(R_{0}\right) \approx \mathbf{R}$.

Proof. It follows from Theorem 1.9 and Theorem 2.5.

Now we shall consider almost finite continuous functions on $X$ taking its values on $\mathbf{Z} \cup\{ \pm \infty\}$. As above we shall write $\mathscr{C}(X, \mathbf{Z} \cup\{ \pm \infty\})$ for the group of all this functions.

LEMMA 2.7. Let $R$ be a regular ring. Let $A$ and $B$ be finitely generated right $R$-modules such that $\operatorname{End}_{R} A$ is unit-regular. If $A / A P \leqq B / B P$ for all prime ideals $P$ of $R$ then $A \lesssim B$.

Proof. In [7, Theorem 4.19] this lemma is proved under the hypothesis of unit-regularity. But, with the notation of [7, Lemma 4.18], it is only necessary that the $R$-module $A_{1} / A_{1} K$ cancels from direct sums, and it is easily seen that this also occurs if $\operatorname{End}_{R} A$ is unit-regular. 
The proof of the next result is quite similar to Theorem 2.5.

THEOREM 2.8. Let $R$ be a regular right self-injective ring of Type $\mathrm{I}_{\infty}$ and let $e_{0}$ be a faithful abelian idempotent in $R$. Then

(i) the rule

$$
[e R] \mapsto \varphi_{e}, \quad \varphi_{e}(M)=d_{M}\left(e R: e_{0} R\right)
$$

defines a partially ordered abelian group isomorphism

$$
\varphi: K_{0}\left(R_{0}\right) \stackrel{\approx}{\rightarrow} \mathscr{C}(X, \mathbf{Z} \cup\{ \pm \infty\})
$$

(ii) the map

$$
L_{2}\left(R_{0}\right) \rightarrow L(\mathscr{C}(X, \mathbf{Z} \cup\{ \pm \infty\})), \quad J \mapsto \varphi\left(K_{0}(J)\right)
$$

is a lattice isomorphism.

Proof. (i) First we prove that if $e$ is an idempotent in $R_{0}$ then $d_{M}\left(e R: e_{0} R\right)$ is either an integer or $\infty$. For this we need only prove that if $n f R \leqslant m g R$, where $m, n$ are positive integers and $f, g$ are idempotents with $g$ abelian, then there exists an integer $s, s \leq m / n$, such that $f R \lesssim s g R$.

Let $P$ be a prime ideal in $R$ and let $\bar{f}, \bar{g} \in R / P$ the images of $f$ and $g$ in $R / P$ respectively. Then $n \bar{f} R / P \leqq m \bar{g} R / P$. Since $R / P$ is prime and $\bar{g}$ is abelian in $R / P$ we see that $\bar{g} R / P$ is a simple module and so $\bar{f} R / P \approx r \bar{g} R / P$ for some $r \in N$. Hence $\bar{f} R / P \leqslant[m / n] \bar{g} R / P$, where $[m / n]$ denotes the integer part of $m / n$. By Lemma 2.7 we obtain $f R \leq[m / n] g R$ as desired.

As in the proof of Theorem 2.5 (i) we derive that $\varphi$ is a well-defined injective map.

Now we are going to prove that $\varphi$ is onto. Like Theorem 2.5 (i) it suffices to prove that for every positive $\alpha$ in $\mathscr{C}(X, \mathbf{Z} \cup\{ \pm \infty\})$ there exists $A$ in $P\left(R_{0}\right)$ such that $\varphi([A])=\alpha$. For each natural $k$, set $X_{k}=$ $\{M \in X \mid \alpha(M)=k\}$. Certainly $X_{k}$ is a clopen set in $X$. Hence $X_{k}=$ $\left\{M \in X \mid e_{k} \notin M\right\}$, for some suitable $e_{k}$ in $B(R)$. Since the $X_{k}$ 's are pairwise disjoint we have that the corresponding $e_{k}$ 's are orthogonal.

For a given natural number $n$, we have, since $R$ is purely infinite, that $n e_{0} R \leqq R$. Thus $\oplus_{k} k e_{k} e_{0} R \leqq R$. Let $A$ denote a principal right ideal of $R$ that is isomorphic to the injective hull of $\oplus_{k} k e_{k} e_{0} R$. There is no difficulty in proving that $A$ belongs to $P\left(R_{0}\right)$. Clearly $e_{k} A \approx k e_{k} e_{0} R$ and, by [7, Proposition 11.3] we have

$$
\varphi([A])(M)=d_{M}\left(A: e_{0} R\right)=d_{M}\left(k e_{k} e_{0} R: e_{0} R\right)=k=\alpha(M),
$$

for all $M \in X_{k}$. Since $\alpha$ is almost finite we see $\varphi([A])=\alpha$.

(ii) It follows similarly to Theorem 2.5 (ii). 
LEMMA 2.9. Let $R$ be a regular right self-injective ring and $I$ an ideal of $R$. If $C \in P(I)$ is purely infinite then $C \approx e R$ for some (purely infinite) idempotent $e$ in $I$.

Proof. Suppose $C=A \oplus B$ for some directly finite right $R$-module $A$ and some purely infinite right $R$-module $B$. Now we prove that $C \approx B$. By [7, Theorem 9.14] there exists $h \in B(R)$ such that $A h \leqq B h$ and $B(1-h) \lesssim A(1-h)$. Then, since $B$ is purely infinite, we have $B(1-h)$ $=0$. So $C(1-h) \approx A(1-h)$ and thus also $A(1-h)=0$. Then $B \leqslant$ $A \oplus B \lesssim B \oplus B \approx B$ and so, by [7, Theorem 10.14] $C=A \oplus B \approx B$.

Now, suppose $C \approx e_{1} R \oplus \cdots \oplus e_{n} R$ for some idempotents $e_{1}, \ldots, e_{n}$ in $I$. By [7, Theorem 10.32] there exists $h_{i} \in B(R)$ such that $h_{i} e_{i} R$ is directly finite and $\left(1-h_{i}\right) e_{i} R$ is purely infinite for $i=1, \ldots, n$. Then by the preceding paragraph we can assume that each $e_{i}$ is purely infinite. Since $R$ satisfies general comparabilaity, there exists $h \in B(R)$ such that $h e_{1} \lesssim h e_{2}$ and $(1-h) e_{2} \lesssim(1-h) e_{1}$. Then it is clear that $e=(1-h) e_{1}$ $+h e_{2}$ is a purely infinite idempotent in $I$ such that $e_{1} R \oplus e_{2} R \approx e R \oplus$ $e R \approx e R$. By induction on $n$ the result follows.

For each ideal $I$ of $R$ we denote by $I_{0}$ the ideal of $R$ generated by all directly finite idempotents in $I$ and by $I_{1}$ the ideal of $R$ generated by all directly finite idempotents in $I$ that are contained in some purely infinite idempotent in $I$.

If $S \in L(\mathscr{C}(X, K))$, where $K$ is either $[-\infty, \infty]$ or $\mathbf{Z} \cup\{ \pm \infty\}$, and $\Gamma$ is a closed set in $X$, then we write $S_{\Gamma}$ for the quotient $S /\{\alpha \in S: \alpha=0$ in some open set in $X$ containing $\Gamma$ \}.

THEOREM 2.10. Let $R$ be a regular right self-injective ring and I an ideal of $R$. Then

(i) $K_{0}(I) \approx K_{0}\left(I_{0}\right) / K_{0}\left(I_{1}\right)$.

(ii) Let $\Gamma(I)=V(\{\mathrm{cc}(g) \mid g$ is a purely infinite idempotent in $I\})$. If $R$ is either Type $\mathrm{II}_{\infty}$ or $\mathrm{I}_{\infty}$ then $K_{0}(I) \approx \varphi\left(K_{0}\left(I_{0}\right)\right)_{\Gamma(I)}$ where $\varphi: K_{0}\left(R_{0}\right) \rightarrow$ $\mathscr{C}(X, K)$ is the map defined in Theorem 2.5 or Theorem 2.8 , respectively.

Proof. (i) First we prove that the natural map $\Psi: K_{0}\left(I_{0}\right) \rightarrow K_{0}(I)$ is onto. Let $A \in P(I)$. By [7, Theorem 10.32] there exists a central idempotent $h$ in $R$ such that $A h$ is directly finite and $A(1-h)$ is purely infinite. Then $[A(1-h)]=0$ in $K_{0}(I)$ and so we can assume that $A$ is directly finite, but in this case it is clear that $A$ belongs to $P\left(I_{0}\right)$.

Now we prove that $\operatorname{Ker} \Psi=K_{0}\left(I_{1}\right)$. Let $A \in P\left(I_{1}\right)$. Since $A$ is isomorphic to a direct sum of principal right ideals, each of which is generated by an idempotent in $I_{1}$, it is clear that in order to prove 
$[A] \in \operatorname{Ker} \Psi$ we may assume $A=e R$ for some idempotent $e$ in $I_{1}$. Then there exists a purely infinite right $R$-module $B$ in $P(I)$ such that $A \leqslant B$. Thus $A \oplus B \approx B$. Then $[A]=0$ in $K_{0}(I)$ and so $K_{0}\left(I_{1}\right) \subseteq \operatorname{Ker} \Psi$.

Conversely, let $[A]-[B] \in \operatorname{Ker} \Psi$. Then by Proposition 1.2 and the proof of Proposition 1.8 (i) there exists a purely infinite right $R$-module $C$ in $P(I)$ such that $A \oplus C \approx B \oplus C$. Now, by the general comparability axiom there exists $h \in B(R)$ such that $B h \leqslant C h$ and $C(1-h) \lessgtr$ $B(1-h)$. Since $B(1-h)$ is directly finite and $C(1-h)$ is purely infinite we see $C(1-h)=0$. From the relation $A \oplus C \approx B \oplus C$ we have $A(1-h) \approx B(1-h)$ so $[A]-[B]=[A h]-[B h]$. Then we may assume $B \leqslant C$ and since $C$ is purely infinite also $A \leqq C$. By Lemma $2.9 A \lesssim$ $e R$ for some purely infinite idempotent $e$ in $I$. Then by Lemma 1.10 $A \subseteq f R$ for some purely infinite idempotent $f$ in $I$. Hence $A \in P\left(I_{1}\right)$. Similarly $B \in P\left(I_{1}\right)$ and then $[A]-[B] \in K_{0}\left(I_{1}\right)$.

(ii) Since $R$ is purely infinite, every element in $K_{0}(I)$ can be written in the form $[e R]-[f R]$ for some idempotents $e, f$ in $I$.

By (i) it suffices to show that $\varphi\left(K_{0}\left(I_{1}\right)\right)=\left\{\alpha \in \varphi\left(K_{0}\left(I_{0}\right)\right): \alpha=0\right.$ in some open set in $X$ containing $\Gamma(I)\}$. If $[e R] \in K_{0}\left(I_{1}\right)$ then there exists a purely infinite idempotent $g$ in $I$ such that $e \leqq g$. Then $e R \oplus g R \approx g R$ and by [7, Theorem 11.11] $d_{M}\left(e R: e_{0} R\right) \leq d_{M}\left(g R: e_{0} R\right)$ for all $M$ in $X$ (here $e_{0}$ is as in Theorem 2.5 or Theorem 2.8). By [7, Proposition 11.3] $d_{M}\left(g R: e_{0} R\right)=0$ if $M \in V(\operatorname{cc}(g))$. So, since $\Gamma(I) \subseteq V(\operatorname{cc}(g))$, we have $\varphi\left(K_{0}\left(I_{1}\right)\right) \subseteq\left\{\alpha \in \varphi\left(K_{0}\left(I_{0}\right)\right) \mid \alpha=0\right.$ in an open set in $X$ containing $\Gamma(I)\}$.

Now we prove the reverse inclusion. For simplicity here we denote by $E$ the set of all purely infinite idempotents in $I$. First we shall note that the set $S=\{\operatorname{cc}(g) \mid g \in E\}$ is an ideal of $B(R)$. If $x \in B(R)$ and $g \in E$, then by [7, Lemma 11.4 (c)] $x \operatorname{cc}(g)=\operatorname{cc}(x g)$. Since $x g \in E$, we see that $x \operatorname{cc}(g) \in S$. Let $g_{1}, g_{2} \in E$ and let $k=\operatorname{cc}\left(g_{1}\right)+\operatorname{cc}\left(g_{2}\right)-2 \operatorname{cc}\left(g_{1}\right) \operatorname{cc}\left(g_{2}\right)$. By [7, Lemma 11.4(c)] and observing that $g_{1}\left(1-\operatorname{cc}\left(g_{2}\right)\right)$ and $g_{2}\left(1-\operatorname{cc}\left(g_{1}\right)\right)$ are orthogonal idempotents we have

$$
\begin{aligned}
\operatorname{cc}\left(g_{1}(1-\right. & \left.\left.\operatorname{cc}\left(g_{2}\right)\right)+g_{2}\left(1-\operatorname{cc}\left(g_{1}\right)\right)\right) \\
& =\operatorname{cc}\left(g_{1}\left(1-\operatorname{cc}\left(g_{2}\right)\right)\right)+\operatorname{cc}\left(g_{2}\left(1-\operatorname{cc}\left(g_{1}\right)\right)\right) \\
& =\operatorname{cc}\left(g_{1}\right)\left(1-\operatorname{cc}\left(g_{2}\right)\right)+\operatorname{cc}\left(g_{2}\right)\left(1-\operatorname{cc}\left(g_{1}\right)\right)=k .
\end{aligned}
$$

By noting that $g_{1}\left(1-\operatorname{cc}\left(g_{2}\right)\right)+g_{2}\left(1-\operatorname{cc}\left(g_{1}\right)\right) \in E$ we obtain that $k \in S$. Then $S$ is an ideal of $B(R)$.

Let $e \in I_{0}$ be an idempotent such that $\varphi([e R])$ is zero in an open set $U$ containing $\Gamma(I)$. For each $M \in \Gamma(I)$ there exists $h_{M} \in B(R)$ with $M \in V\left(h_{M}\right) \subseteq U$. Since $\Gamma(I)$ is compact we can find $M_{1}, \ldots, M_{r} \in \Gamma(I)$ 
with $\Gamma(I) \subseteq V\left(h_{M_{1}}\right) \cup \cdots \cup V\left(h_{M_{r}}\right)=V\left(h_{M_{1}} \cdots h_{M_{r}}\right) ;$ set $h=$ $h_{M_{1}} \cdots h_{M_{r}}$, then from the inclusion $V(S)=\Gamma(I) \subseteq V(h)$ we obtain $h \in S$ and so $h=\operatorname{cc}(g)$ for some $g$ in $E$.

Let $M \in X$. If $1-h \in M$, then by [7, Proposition 11.3 (a)]

$$
d_{M}\left(e(1-h) R: e_{0} R\right)=0 .
$$

If $h \in M$ then, since $V(h) \subseteq U, d_{M}\left(e(1-h) R: e_{0} R\right)=\varphi([e R])(M)=0$. Hence, by [7, Proposition 11.6], $e(1-h)=0$. Let $t \in B(R)$ such that $t e \leqslant t g$ and $(1-t) g \leqslant(1-t) e$. Because $(1-t) e$ is directly finite and $(1-t) g$ is purely infinite, we obtain $(1-t) g=0$ and so $h=\operatorname{cc}(g) \leq t$. Then by multiplying the relation $t e \leqslant g$ by $h$, we obtain $h t e \leqslant h g=g$, and, because $h t=h$ and $h e=e$, we have $e \leq g$. By Lemma 1.10 we may assume $e \leq g$ and so $[e R] \in K_{0}\left(I_{1}\right)$ as desired.

3. Rickart $C^{*}$-algebras. Recall that a $C^{*}$-algebra $A$ is said to be Rickart if the right annihilator of each element in $A$ is generated by a projection. In notation $r(a)=e A$ where $e=e^{2}=e^{*}$. If the annihilator condition holds for every subset of $A$, then $A$ is called an $A W^{*}$-algebra. As usual we shall write $\operatorname{RP}(a)$ (the right projection of $a$ ) for $1-e$. The left projection of $a, L P(a)$, is defined similarly. It is known [3, Proposition 1.3.7 and Lemma 1.8.2] that with the relation $\leq$ the set of all projections of a Rickart $C^{*}$-algebra is a complemented $\chi_{0}$-complete lattice. Two projections $e$ and $f$ are said to be equivalent, written $e \sim f$, if $e A \approx f A$. A projection $e$ is said to be finite if $e \sim f \leq e$ implies $e=f$. We say $A$ is finite if 1 is a finite projection. Since $A$ is a $C^{*}$-algebra $e \sim f$ if and only if $e$ and $f$ are *-equivalent, that is $e=x x^{*}$ and $f=x^{*} x$ for some $x \in e A f$ cf. [9, Proposition 19.1 (a)]. If $e$ is an idempotent of a $C^{*}$-algebra $A$, then there exists a unique projection $f$ in $A$ such that $e A=f A$ cf. [9, proof of Proposition 19.1 (b)]. From this we see that Rickart $C^{*}$-algebras are precisely those $C^{*}$-algebras that are principal projective. It seems to be unknown whether Rickart $C^{*}$-algebras are semihereditary.

For background and basic concepts on Rickart $C^{*}$-algebras the reader can consult [3].

Proposition 3.1. If $A$ is a Rickart $C^{*}$-algebra and $I$ is an ideal of $A$ then $K_{0}(I)=G(I)=K_{0}(\bar{I})$, where $\bar{I}$ is the closure of $I$.

Proof. Let $E$ be the set of all projections in $I$. It follows from [3, Proposition 5.22.1] that the sub- $C^{*}$-algebras $\{e A e+\mathrm{C} 1\}_{e \in E}$ form a directed system. Since $\bar{I}$ is the closed $\mathbf{C}$-linear span of its projections $[3, \mathrm{p}$. 
142, Exc. 7A] we have that $C^{*}$-dir. $\lim _{e \in E}(e A e+\mathbf{C} 1)=\bar{I}+\mathbf{C} 1$. Now it follows from [9, Proposition 19.9] that the natural map

$$
\underset{e \in E}{\operatorname{dir} . \lim _{0}} K_{0}(e A e+\mathbf{C}) \rightarrow K_{0}(\bar{I}+\mathbf{C})
$$

is a group isomorphism. Since the diagram

$$
\begin{array}{cc}
\underset{e \in E}{\operatorname{dir} . \lim _{0}} K_{0}(e A e+\mathbf{C}) & \rightarrow \quad K_{0}(I+\mathbf{C}) \\
& i \downarrow \\
K_{0}(\bar{I}+\mathbf{C}) &
\end{array}
$$

is commutative, where the maps are the natural ones, then the map

$$
\underset{e \in E}{\operatorname{dir} . \lim _{0}} K_{0}(e A e+\mathbf{C}) \rightarrow K_{0}(I+\mathbf{C})
$$

is injective, and onto by [9, Proposition 19.3].

Thus, by Proposition 0.1 we have $K_{0}(I)=K_{0}(\bar{I})=G(I)$.

Let $A$ be a $C^{*}$-algebra and let $I$ be an ideal of $A$. If $\pi: A \rightarrow A / I$ is the natural surjection, then we set $\mathscr{F}(I, A)=\pi^{-1}(U(A / I))$. An element of $\mathscr{F}(I, A)$ is said to be a Fredholm element of $A$ relative to $I$. In the case where $A=B(H)$ is the ring of all bounded operators on a separable Hilbert space and $I=\mathscr{K}$ is the ideal of compact operators, then the elements of $\mathscr{F}(\mathscr{K}, B(H))$ are the usual Fredholm operators cf. [6, Chapter $5]$.

Let us recall briefly some basic results on index theory for Fredholm operators. If $T \in \mathscr{F}(\mathscr{K}, B(H))$, then by Atkinson's theorem [6, 5.17 Theorem] $\operatorname{dim} \operatorname{ker} T$ and $\operatorname{dim} \operatorname{Ker} T^{*}$ are both finite and the map $i$ : $\mathscr{F}(\mathscr{K}, B(H)) \rightarrow \mathbf{Z}$ given by $T \mapsto \operatorname{dim} \operatorname{Ker} T^{*}$-dim $\operatorname{Ker} T$ (the index map) is a continuous monoid homomorphism [6, 5.36 Theorem]. Furthermore the connected components of $\mathscr{F}(\mathscr{K}, B(H))$ are $i^{-1}(n), n \in \mathbf{Z}[\mathbf{6}, 5.36$ Theorem]. Breuer [4] [5] generalizes this result to an arbitrary $W^{*}$-algebra (here the compact ideal means the closure of the ideal generated by all finite projections in $A$ ). More recently Olsen [15] has defined an index map for each closed ideal $I$ of a $W^{*}$-algebra which permits to describe the connected components of $\mathscr{F}(I, A)$.

Next we shall extend Breuer's theory to arbitrary Rickart $C^{*}$-algebras. In order to obtain an explicit index map for any closed ideal in a Rickart $C^{*}$-algebra $A$ we will need the following additional axioms on $A$ :

(i) $A$ has a projection $e$ such that $e \sim 1-e \sim 1$

(ii) $A$ satisfies the general comparability axiom (i.e. for each pair of projections $e, f$ there exists a central projection $h$ such that $h e \lesssim h f$ and $h(1-f) \lesssim h(1-e))$. 
As we shall see this axioms are not an obstacle for constructing an index theory for arbitrary $A W^{*}$-algebras.

The following lemma is known under the additional hypothesis of general comparability (see [3, Lemma 1.8.3, Theorem 3.17.3]).

If $A$ is a Rickart $C^{*}$-algebra, then we denote by $\mathscr{K}=\mathscr{K}(A)$ the closure of the ideal generated by all finite projections of $A$. We say that $\mathscr{K}$ is the compact ideal of $A$.

\section{LEMMA 3.2. Every projection in $\mathscr{K}$ is finite.}

Proof. Let $I$ be the ideal generated by all finite projections in $A$.

Since $\mathscr{K}$ is the closure of $I$ it is well-known that every projection in $\mathscr{K}$ belongs to $I$ cf [3, Chapter $5 \$ 22$ Exercise 6A]. Now let $f$ be a projection in $I$, then $f=\sum x_{i} e_{i} y_{i}$, where $x_{i}, y_{i} \in A$ and the $e_{i}$ 's are finite projections. Consider now the map $\psi: \oplus e_{i} A \rightarrow f A$ defined by $\psi\left(\sum e_{i} r_{i}\right)=$ $\sum f x_{i} e_{i} r_{i}$. Clearly $\psi$ is an onto $A$-module homomorphism. Thus $f A \lesssim$ $\oplus e_{i} A$. Now a finite Rickart $C^{*}$-algebra has stable range $1 \mathrm{cf}[10]$. So the endomorphisms rings $e_{i} A e_{i} \approx \operatorname{End}_{R}\left(e_{i} A\right)$ have stable range 1 . In particular $\oplus e_{i} A$ cancels from direct sums of right $A$-modules and, since $f A$ is isomorphic to a direct summand of $\oplus e_{i} A$, the same is true for $f A$. Therefore $f$ is finite.

If $M$ and $N$ are right $A$-modules, then $M \hookrightarrow N$ means that $M$ is isomorphic to a submodule of $N$.

LEMMA 3.3. If $A$ is a Rickart $C^{*}$-algebra, then

(i) If $e \in A$ is a finite projection, then $e A$ does not contain an infinite direct sum of nonzero pairwise isomorphic right ideals. In particular, every $A$-module $M \hookrightarrow e A$ is directly finite.

(ii) If $P$ and $Q$ are directly finite cyclic projective right $A$-modules such that $P \hookrightarrow Q$ and $Q \hookrightarrow P$, then $P \approx Q$.

(iii) If $x$ is an element of $A$ such that $\operatorname{LP}(x)$ is finite, then $\operatorname{LP}(x) \sim$ $\mathrm{RP}(x)$. Further $x A \approx x^{*} A$.

Proof. (i) Let $\left\{A_{n}\right\}$ be a sequence of pairwise isomorphic right ideals contained in $e A$. Then $\left\{A_{n} e\right\}$ is a sequence of pairwise isomorphic right ideals of $e A e$. Now $e A e$ is a finite Rickart $C^{*}$-algebra and so, $R$, its classical ring of quotients [1, Theorem 3.1(i)] [11, Theorem 2.1] is an $\boldsymbol{\aleph}_{0}$-continuous regular ring which contains an infinite direct sum of pairwise isomorphic right ideals. By [8, Proposition 1.1] $A_{i} e \otimes_{e A e} R=0$, hence $A_{i} e=0$. But $A$ is semiprime so $0=e A_{i}=A_{i}$ as desired. 
(ii) We may assume $P=e A$ and $Q=f A$ for some finite projections $e$, $f$ in $A$. Let $g$ be the supremum of $e$ and $f$. By [3, Proposition 5.22.1] $g \in \mathscr{K}$ and it follows from Lemma 3.2 that $g$ is finite and so $g A g$ is a finite Rickart $C^{*}$-algebra. Now $e(g A g) \hookrightarrow f(g A g)$ and $f(g A g) \hookrightarrow e(g A g)$. If $R$ is the classical ring of quotients of $g A g$, then because $R$ is regular we have $e R \leqq f R$ and $f R \leqq e R$. But $R$ is unit-regular cf [11, Theorem 3.2] so $e R \approx f R$. Because of the unit regularity one has [7, Corollary 4.23] that $e R$ and $f R$ are perspective in the lattice $L(R)$ of principal right ideals of $R$. By [11, Theorem 2.1(3)] $L(R)=L(g A g)$ so that $e \sim f$ in $g A g$ and so in A.

(iii) Since $x A \approx \operatorname{RP}(x) A$ and $x A \leq \mathrm{LP}(x) A$, we see that $\mathrm{RP}(x) \hookrightarrow$ $\mathrm{LP}(x)$, similarly $\mathrm{LP}(x) \hookrightarrow \mathrm{RP}(x)$. $\mathrm{By}$ (i) $\mathrm{RP}(x)$ is finite and then from (ii) we get $\mathrm{LP}(x) \sim \operatorname{RP}(x)$ and $x A \approx x^{*} A$.

LEMMA 3.4. Let e be a finite projection in a Rickart $C^{*}$-algebra A. If $x \in A$ is such that $x x^{*}$ and e commute then

$$
x A \cap e A \approx e x x^{*} A \approx e\left(x x^{*}\right)^{1 / 2} A .
$$

Proof. Since $\mathrm{LP}(e x) \leq e$ we see that $\mathrm{LP}(e x)$ is a finite projection. By Lemma 3.3 (iii) $e x A \approx x^{*} e A$. Since $r\left(x^{*} e\right)=r\left(x x^{*} e\right)$ and $x x^{*}$ commutes with $e$ we have $x A \cap e A \subseteq e x A \approx e x x^{*} A \subseteq x A \cap e A$. By Lemma 3.3(i), $x A \cap e A$ and $e x x^{*} A$ are directly finite right $A$-modules. Moreover, left multiplication by $x$ induces an epimorphism from $r((1-e) x)$ to $x A \cap e A$, then $x A \cap e A$ is a cyclic right ideal and so projective. Thus by Lemma 3.2 (ii) $e x x^{*} A \approx x A \cap e A$. Since $r\left(e x x^{*}\right)=r\left(e\left(x x^{*}\right)^{1 / 2}\right)$ left multiplication by $\left(x x^{*}\right)^{1 / 2}$ gives $\left(e x x^{*}\right) A \approx e\left(x x^{*}\right)^{1 / 2} A$.

Notice that if $A$ has polar decomposition, then by [3, Proposition 4.21.3] $x A=\left(x x^{*}\right)^{1 / 2} A$ for every $x$ in $A$. Thus in this case the preceding lemma is obvious. It is not known whether Rickart $C^{*}$-algebras have polar decomposition cf. [3, Chapter $4 \S 21$ Exercise 10D]. In fact we have the following result noted by Handelman.

LEMMA 3.5 (Handelman). A semihereditary Rickart C*-algebra has polar decomposition.

Proof. If $A$ is a semihereditary Rickart $C^{*}$-algebra, then $M_{2}(A)$ is also a Rickart $C^{*}$-algebra cf. [9, Theorem 7.4, Proof of Proposition 19.1 (b)]. Now $M_{2}(A)$ contains two orthogonal copies of $A$ and by using the same techniques than in the proof of [3, Proposition 4.20.2] we see that partial isometries are $\boldsymbol{\aleph}_{0}$-addable in $A$. But then, as it is noted in $[3$, p. 276 Exercise 11 (ii)] $A$ has polar decomposition. 
LeMMa 3.6. Let $A$ be a Rickart $C^{*}$-algebra and let $I$ be an ideal of $A$. If $x$ is an element of $A$ then the following are equivalent

(i) $x \in \mathscr{F}(I, A)$

(ii) There exist a positive unit $\gamma$ and projections $e$, $f$ in I such that

$$
\begin{aligned}
e \gamma x x^{*} \gamma & =\gamma x x^{*} \gamma e \\
(1-e) \gamma x x^{*} \gamma(1-e) & =1-e \\
x^{*} \gamma(1-e) \gamma x & =1-f .
\end{aligned}
$$

(iii) There exist projections $f, g$ in $I$ such that $1-f \in x^{*} A$ and $1-g \in x A$.

Moreover, if either $I \subseteq \mathscr{K}$ or $A$ is semihereditary, then for any pair of projections $e, f$ satisfying (ii) we have $r\left(x^{*}\right) \oplus f A \approx r(x) \oplus e A$.

Proof. (i) $\Rightarrow$ (ii). If $x \in \mathscr{F}(I, A)$ then $x A+z A=A$ for some $z \in I$ and, since $A$ is a $C^{*}$-algebra, $x x^{*}+z z^{*}$ is a unit. By [3, Proposition 1.8.4], for a given $\varepsilon>0$, there exists a projection $p \in z z^{*} A$ with $\| z z^{*}-$ $p z z^{*} \|<\varepsilon$. Thus we can choose $p$ such that $x x^{*}+p z z^{*}$ is a unit. But then $x A+p A=A$, say $x x^{*}+p=\left(\gamma^{-1}\right)^{2}$ where $\gamma=\gamma^{*}$ is a unit. Define $e=\mathrm{LP}(\gamma p \gamma)$, since $\gamma p \gamma$ is positive $e=\operatorname{RP}(\gamma p \gamma)$, moreover $e \in I$. Since $\gamma x x^{*} \gamma+\gamma p \gamma=1$ we see that $e$ commutes with $\gamma x x^{*} \gamma$. By multiplying the latter relation by $1-e$ we get $(1-e) \gamma x x^{*} \gamma(1-e)=1-e$. Therefore $x^{*} \gamma(1-e) \gamma x$ is a projection, say $1-f$. Since $x \in \mathscr{F}(I, A)$, we see that $f \in I$. The proof is complete.

(ii) $\Rightarrow$ (iii) Since $e \gamma x x^{*}=\gamma x x^{*} \gamma e$ and $(1-e) \gamma x x^{*} \gamma(1-e)=1-e$, we see that $1-e \in \gamma x A$, that is $\gamma^{-1}(1-e) \gamma \in x A$. Now $\gamma^{-1}(1-e) \gamma A$ $=(1-g) A$, where $g$ is a projection, and because $e \in I$ we see that $g \in I$ cf. [9, proof of Proposition 19.1 (b)]. Hence $1-g \in x A$. On the other hand is clear that $1-f \in x^{*} A$.

Obviously (iii) implies (i).

Suppose now that $e$ and $f$ are projections satisfying (ii). Since $r(x)=r(\gamma x)$ and $r\left(x^{*}\right) \approx r\left(x^{*} \gamma\right)$ we may assume, without loss of generality, that $\gamma=1$. Now consider the following exact sequences

$$
\begin{aligned}
& 0 \rightarrow r(x) \rightarrow r((1-e) x) \rightarrow x A \cap e A \rightarrow 0 \\
& 0 \rightarrow r\left(x^{*}\right) \rightarrow r\left((1-e)\left(x x^{*}\right)^{1 / 2}\right) \rightarrow\left(x x^{*}\right)^{1 / 2} A \cap e A \rightarrow 0
\end{aligned}
$$

If $I \subseteq \mathscr{K}$, then, by Lemma 3.4, $x A \cap e A \approx\left(x x^{*}\right)^{1 / 2} A \cap e A$. In the case where $A$ is semihereditary we also have this isomorphism because then $A$ has polar decomposition (Lemma 3.5). Thus in both cases we can apply Schanuel's lemma to get

$$
r(x) \oplus r\left((1-e) x x^{*}\right) \approx r\left(x^{*}\right) \oplus r((1-e) x),
$$


now

$$
r\left((1-e) x x^{*}\right)=r\left((1-e) x x^{*}(1-e)\right)=r\left(x^{*}(1-e)\right)=e A
$$

and $r((1-e) x)=f A$. The proof is complete.

Proposition 3.7. Let $A$ be a Rickart $C^{*}$-algebra and let I be an ideal of $A$. If $\alpha$ denotes the composite map

$$
\mathscr{F}(I, A) \rightarrow U(A / I) \rightarrow K_{1}(A / I) \stackrel{\delta}{\rightarrow} K_{0}(I)
$$

then we have

(i) If $I \subseteq \mathscr{K}$ then

$$
\alpha(x)=\left[r\left(x^{*}\right)\right]-[r(x)]
$$

and $\mathrm{LP}(x) \sim \operatorname{RP}(x)$ for all $x \in \mathscr{F}(I, A)$.

(ii) If $A$ is semihereditary, then

$$
\alpha(x)=\left[r\left(x^{*}\right)\right]-[r(x)] \text { for all } x \in \mathscr{F}(I, A) .
$$

Proof. Let $\beta: \mathscr{F}(I, A) \rightarrow K_{0}(I)$ be the map defined by $\beta(x)=$ $\left[r\left(x^{*}\right)\right]-[r(x)]$. Then we must prove that $\beta=\alpha$.

Let $x \in \mathscr{F}(I, A)$. Now let $\gamma, e, f$ as in Lemma 3.6 (ii). Then we have $\beta(\gamma x)=\left[r\left(x^{*} \gamma\right)\right]-[r(\gamma x)]=\left[r\left(x^{*}\right)\right]-[r(x)]=\beta(x)$. On the other hand it is clear that $\alpha(\gamma)=0$ so $\alpha(\gamma x)=\alpha(\gamma)+\alpha(x)=\alpha(x)$. Hence we may assume $\gamma=1$. For simplicity we shall write $y=(1-e) x$, then we have

$$
\begin{aligned}
& y y^{*}=1-e \\
& y^{*} y=1-f .
\end{aligned}
$$

It follows from Lemma 0.2 and the remarks preceding it that

$$
\begin{aligned}
\alpha(y) & =[(0, e) D]-[(0, f) D] \in K_{0}(I, A) \\
& =[e A]-[f A] \in K_{0}(I)
\end{aligned}
$$

Hence

$$
\alpha(x)=\alpha(y)=[e A]-[f A]=\left[r\left(x^{*}\right)\right]-[r(x)]=\beta(x) .
$$

Suppose now $I \subseteq \mathscr{K}$. Then

$$
1-e=\operatorname{LP}(y)=\operatorname{LP}((1-e) x) \sim 1-f=\operatorname{RP}(y)=\operatorname{RP}((1-e) x)
$$

and, by Lemma 3.3 (iii), we obtain

$$
e \geq \operatorname{LP}(e x) \sim \operatorname{RP}(e x) .
$$


Since $e x x^{*}=x x^{*} e$ we then get

$\mathrm{LP}(x)=\mathrm{LP}((1-e) x)+\mathrm{LP}(e x) \sim \mathrm{RP}((1-e) x)+\mathrm{RP}(e x) \leq \mathrm{RP}(x)$,

so $\mathrm{LP}(x) \lesssim \operatorname{RP}(x)$, for all $x \in \mathscr{F}(I, A)$. By symmetry $\mathrm{RP}(x) \lesssim \operatorname{LP}(x)$. Now it follows from the generalized Schröder-Bernstein theorem that $\operatorname{RP}(x) \sim \operatorname{LP}(x)$.

Corollary 3.8. If $A$ is a semihereditary Rickart $C^{*}$-algebra and I is an ideal of $A$, then the connecting map

$$
\delta: K_{1}(A / I) \rightarrow K_{0}(I)
$$

is defined by

$$
\delta(\bar{X})=\left[r\left(x^{*}\right)\right]-[r(X)]
$$

where $X$ is any matrix over $A$ such that modulo $I$ is an invertible matrix representing $\bar{X} \in K_{1}(A / I)$.

Proof. Since $A$ is semihereditary, matrix rings over $A$ are also semihereditary Rickart $C^{*}$-algebras. The result follows, by using matrices, as in the proof of Proposition 3.7 (ii).

Theorem 3.9. Let $A$ be a Rickart $C^{*}$-algebra and let I be a closed ideal in $A$ consisting of compact elements. Then

(i) Let $\pi: \mathscr{F}(I, A) \rightarrow U(A / I)$ be the natrual surjection and let $\lambda$ be the composite map

$$
U(A / I) \rightarrow K_{1}(A / I) \stackrel{\delta}{\rightarrow} K_{0}(I) .
$$

Denote by $U(A / I)^{0}$ the connected component of $1 \in U(A / I)$. Then

$$
U(A / I)^{0}=\pi(U(A))=\operatorname{ker} \lambda .
$$

(ii) If $K_{0}(I)$ is considered as a discrete group, then the map

$$
\begin{aligned}
\alpha: \mathscr{F}(I, A) & \rightarrow K_{0}(I) \\
x & \rightarrow\left[r\left(x^{*}\right)\right]-[r(x)]
\end{aligned}
$$

is a continuous monoid homomorphism.

(iii) $\alpha(\mathscr{F}(I, A))$ consists of those elements $z \in K_{0}(I)$ such that $z=$ $[e A]-[f A]$ where $e$ and $f$ are projections in $I$ with $1-e \sim 1-f$. Moreover, two projections $e, f$ in I satisfy $[e A]=[f A] \in K_{0}(I)$ if and only if $e \sim f$. 
(iv) $x, y \in \mathscr{F}(I, A)$ lie in the same connected component if and only if $\alpha(x)=\alpha(y)$. Further $\alpha$ induces a group isomorphism

$$
U(A / I) / U(A / I)^{0} \approx \alpha(\mathscr{F}(I, A))
$$

(v) $\alpha(x)=0$ if and only if $\mathrm{LP}(x)$ and $\mathrm{RP}(x)$ are unitary equivalent.

(vi) $\alpha(x)=0$ if and only if $x+$ I contains a unit.

Proof. Consider any $x \in \mathscr{F}(I, A)$. Say $e A=r\left(x^{*}\right)$ and $f A=r(x)$, where $e$ and $f$ are projections which belong to $I$. By Proposition 3.7 (i) $1-e=\operatorname{LP}(x) \sim \operatorname{RP}(x)=1-f$. Conversely let $z=[e A]-[f A]$ with $1-e \sim 1-f$. Suppose $x \in A$ is such that $x x^{*}=1-e, x^{*} x=1-f$. Certainly $x \in \mathscr{F}(I, A)$ and $r\left(x^{*}\right)=e A, r(x)=f A$. Therefore $\alpha(x)=z$.

Suppose now $[e A]=[f A] \in K_{0}(I)$. If for each projection $g$ we write $A_{g}=g A g+\mathrm{C}$, then $I+\mathrm{C}$ is the $C^{*}$-direct limit of the $A_{g}$ 's for $g$ in $I$. By [9, Theorem 19.9] $K_{0}(I+\mathrm{C} \cdot 1)=\operatorname{dir} \cdot \lim . K_{0}(g A g+\mathrm{C})$, so $K_{0}(I)=$ dir.lim. $K_{0}(g A g)$. By Proposition $3.1 K_{0}(g A g)=G(g A g)$, then there exists a projection $g$ in $I$ with $e, f \leq g$ and a finitely generated projective $A_{g}$-module $C$ such that $e A_{g} \oplus C \approx f A_{g} \oplus C$.

Since $A_{g}$ has stable range $1, C$ cancels from the direct sums and so $e A_{g} \approx f A_{g}$. Therefore $e \sim f$. Thus (iii) follows.

(i) Now we compute $\operatorname{Ker} \lambda$. If $x \in F(I, A)$ then we shall denote $\pi(x)$ by $\bar{x}$. Note that $\pi(U(A)) \subset \operatorname{Ker} \lambda$. Conversely, if $\lambda(\bar{x})=0$, then by (iii) $r\left(x^{*}\right) \approx r(x)$ and with the notation of Lemma 3.6 we have

$$
\begin{aligned}
(1-e) \gamma x x^{*} \gamma(1-e) & =1-e \\
x^{*} \gamma(1-e) \gamma x & =1-f
\end{aligned}
$$

and $e \sim f$. Let $u$ be a unitary such that $f=u e u^{*}$. Then it is easily seen that

$$
\begin{aligned}
& \left((1-e) \gamma x+u^{*} f\right)\left(x^{*} \gamma(1-e)+f u\right)=1 \\
& \left(x^{*} \gamma(1-e)+f u\right)\left((1-e) \gamma x+u^{*} f\right)=1
\end{aligned}
$$

so $(1-e) \gamma x+u^{*} f \in U(A)$. Hence $\gamma x-\left(e \gamma x+u^{*} f\right) \in U(A)$. Putting $i=\gamma^{-1}\left(e \gamma x+u^{*} f\right) \in I$ we have that $x-i \in U(A)$ and so $\bar{x} \in \pi(U(A))$. Since the unit group of a Rickart $C^{*}$-algebra is connected $\pi(U(A))$ also is. If we prove that $\pi(U(A))$ is open, then it is clear that $\pi(U(A))=$ $U(A / I)^{0}$. For this let $\bar{u} \in \pi(U(A))$ such that $\|\bar{u}-\overline{1}\|<1$. This means that $\inf _{i \in I}\|(u+i)-1\|<1$. Thus there exists $i \in I$ with $\|(u+i)-1\|$ $<1$, then $u+i$ is a unit and therefore $\bar{u} \in \pi(U(A))$. 
By Proposition 3.7 (i) $\alpha=\lambda \pi$. So (ii) and the isomorphism $U(A / I) U(A / I)^{0} \approx \alpha(F(I, A))$ of (iv) follow. In order to end the proof of (iv) note that $\alpha(x)=\alpha(y)$ if and only if $\bar{x}$ and $\bar{y}$ lie in the same connected component of $U(A / I)$. Since the map $\pi$ is open and onto the result follows.

(v) Suppose $\alpha(x)=0$, then by (iii) $r(x) \approx r\left(x^{*}\right)$ and since $\operatorname{LP}(x) \sim$ $\operatorname{RP}(x)$ we see that $\operatorname{LP}(x)$ and $\operatorname{RP}(x)$ are unitary equivalent.

(vi) By (i) it is clear that $\alpha(x)=0$ if and only if $\bar{x} \in \pi(U(A))$. So $\alpha(x)=0$ if and only if $x+I$ contains a unit.

LEMMA 3.10. Let $M$ be a $2 \times 2$ matrix over a ring $R$. If for some entry $a$ in $M$ there exist $b, c$ in $R$ such that bac $=1$, then $M$ can be reduced by elementary transformations to a diagonal matrix.

Proof. There is no loss of generality in assuming that $M$ is of the form

$$
M=\left(\begin{array}{ll}
* & a \\
* & *
\end{array}\right)
$$

and $b a c=1$. Now notice that the matrices

$$
P=\left(\begin{array}{cc}
b & 0 \\
1-a c b & a c
\end{array}\right) \text { and } Q=\left(\begin{array}{cc}
b a & 0 \\
1-c b a & c
\end{array}\right)
$$

belong to $G E_{2}(R)$. But then we have that $P M Q$ is of the form

$$
\left(\begin{array}{ll}
* & 1 \\
* & *
\end{array}\right),
$$

since this matrix can be reduced to a diagonal one, the same holds for M.

Proposition 3.11. Let $A$ be a Banach algebra satisfying the following condition:

For each $a \in A$ and $\varepsilon>0$ there exists an idempotent $e \in a A$ and $a$ central idempotent $h \in A$ such that

(a) $\|a-e a\|<\varepsilon$

(b) he $\sim h$ and $(1-h)(1-e) \sim(1-h)$. Then $A$ is $a G E_{2}$-ring.

Proof. For any Banach algebra [16, Proposition 8.7] we have $\mathrm{GL}_{2}(A)^{0}$ $\subseteq G E_{2}(A)$. Hence $G E_{2}(A)$ is clopen. In order to prove that $G E_{2}(A)=$ $\mathrm{GL}_{2}(A)$ it suffices to note that $G E_{2}(A)$ is a dense subset of $\mathrm{GL}_{2}(A)$. For this let

$$
X=\left(\begin{array}{ll}
a & b \\
c & d
\end{array}\right) \in \mathrm{GL}_{2}(A)
$$


and $\varepsilon>0$. Choose an integer $n$ such that $n>1 / \varepsilon,\left\|X^{-1}\right\|$. By hypothesis there exist idempotents $e$ and $h$, with $h$ central, such that

$$
\|a-e a\|<1 / n
$$

and

$$
h e \sim h \text { while }(1-h)(1-e) \sim 1-h .
$$

Consider now the matrix

$$
M=\left(\begin{array}{cc}
e a & b \\
c & d
\end{array}\right)
$$

Then we have $\|X-M\|<1 / n<1 /\left\|X^{-1}\right\|$. Therefore $M \in \mathrm{GL}_{2}(A)$. We claim that $M \in G E_{2}(A)$. Since $h$ induces a ring decomposition of $A$, by cutting down to each part we may assume that either (i) $e \sim 1$ or (ii) $1-e \sim 1$. In the first case there exist $x, y \in A$ such that $x e y=1$ and since $e=a z$, for some $z \in A$, we have $x(e a) z y=1$. It follows from Lemma 3.10 that $M \in G E_{2}(A)$.

Now suppose that $1-e \sim 1$. From the relation $e A+b A=A$ we see that $1-e \in(1-e) b A$. Hence $x b y=1$, for some $x, y \in A$. The result follows again by using Lemma 3.10 .

We say that a Rickart $C^{*}$-algebra $A$ is purely infinite if 1 is the supremum of a sequence of orthogonal projections all equivalent to 1 . It is a simple exercise to see that $A$ is purely infinite if and only if $A \approx A^{2}$ as right $A$-modules.

LEMMA 3.12 (Pere Ara). Let $A$ be a purely infinite Rickart $C^{*}$-algebra satisfying general comparability. Suppose e is a projection such that $e \sim 1-$ $e$, then $e \sim 1$.

Proof. Denote by $\vee$ and $\wedge$ the operations of taking supremum and infimum respectively. Since $A$ is purely infinite choose a projection $f$ such that $f \sim 1-f \sim 1$. Define

$$
\begin{aligned}
& g=(1-e) \wedge f \\
& h=L P(e f) \quad(=(1-e) \vee f-(1-e)) .
\end{aligned}
$$

Since $A$ satisfies the parallelogram law [3, Theorem 2.13.1]

$$
\begin{aligned}
h A \oplus g A & =((1-e) \vee f-(1-e)) A \oplus((1-e) \wedge f) A \\
& \approx(f-(1-e) \wedge f) A \oplus((1-e) \wedge f) A \\
& =f A .
\end{aligned}
$$


Since $h<e$ and $g<1-e$ we see that $e-h$ and $(1-e)-g$ are orthogonal projections, we have

$$
\begin{aligned}
(e-h) A & \oplus((1-e)-g) A \\
& =(e-h) A \oplus((1-e)-(1-e) \wedge f) A \\
& \approx(e-h) A \oplus((1-e) \vee f-f) A \\
& =(e-h) A \oplus(h+1-e-f) A=(1-f) A .
\end{aligned}
$$

Now we shall prove that $e \sim 1$. Since $A$ satisfies general comparability we may assume that either $g \leqslant e-h$ or $e-h \leqslant g$. In the first case we have (by using (1)) that

$$
1 \sim f \sim h+g \leq h+(e-h)=e \leq 1,
$$

while in the second case we have (by using (2)) that

$$
\begin{aligned}
1 & \sim 1-f \sim(e-h)+((1-e)-g) \leq g+((1-e)-g) \\
& =1-e \sim e \leq 1 .
\end{aligned}
$$

Thus in both cases we see that $1 \leq e \leq 1$. Then the generalized Schröder-Bernstein theorem yields the result.

THEOREM 3.13. Let $A$ be a purely infinite Rickart $C^{*}$-algebra satisfying general comparability. If $I$ is an ideal of $A$, then

(i) $K_{1}(A / I)=U(A / I) / \pi(U(A))=U(A / I)^{\mathrm{ab}}$.

(ii) If I is closed in $A$, then

$$
\pi(U(A))=U(A / I)^{0} .
$$

(iii) $A / I$ is a $G E$-ring.

Proof. (i) Since $A^{2} \approx A$ we have $(A / I)^{2} \approx A / I$ as $A / I$-modules. In order to prove that $K_{1}(A / I)=U(A / I)^{\mathrm{ab}}$ it suffices to show cf. [13, Theorem 1.2 (iii)] that $A / I$ is a $G E_{2}$-ring. In proving this we first assume that $I$ is closed. By noting that the hypotheses in Proposition 3.11 carry over algebra Banach factors, it suffices to verify that the algebra $A$ satisfies (a) and (b) of that proposition. Obviously (a) is an immediate consequence of the spectral theorem [3, Proposition 1.8.4]. For (b), let $e$ be an idempotent in $A$. By general comparability there exists a central idempotent $h$ such that $h(1-e) \leqslant h e(1)$ and $(1-h) e \leqslant(1-h)(1-e)$ (2). From the relation (1) we have $h A \lesssim(h e A)^{2}$. Since $A$ is purely infinite we have also $(h e A)^{2} \leq h A$. So $h A \approx(h e A)^{2}$ and we can write $h A=e_{1} A$ $\oplus e_{2} A$ for some projections $e_{1}, e_{2} \in h A$ such that $e_{1} \sim e_{2} \sim h e$. Then $e_{1} \sim h-e_{1}$ and Lemma 3.12 yields $e_{1} \sim h$ so $h e \sim h$. Using the relation (2) we have $(1-h)(1-e) \sim 1-h$. Thus we have shown that $A / I$ is a $G E_{2}$-ring for any closed ideal $I$ of $A$. Now assume $I$ is an arbitrary ideal of $A$. Let $M \in M_{2}(A)$ such that $M$ is a unit modulo $I$. If $\bar{I}$ denotes the 
closure of $I$ in $A$, then $M$ is a unit modulo $\bar{I}$ and by the above we may assume, by using elementary transformations, that $M$ is of the form

$$
\left(\begin{array}{ll}
u & 0 \\
0 & *
\end{array}\right)
$$

where $u+\bar{I}$ is a unit of $A / \bar{I}$. It is easily seen that $u+I$ must be a unit of $A / I$. Now by elementary transformations we can reduce $M$ modulo $I$ to obtain a diagonal matrix. Thus $A / I$ is a $G E_{2}$-ring. If $A$ is a purely infinite Rickart $C^{*}$-algebra then $A \approx M_{2}(A)$ and so $A$ is semihereditary. In particular, by Lemma 3.5, $A$ has polar decomposition.

Now by using that $U(A)$ is a perfect group [13, proof of Theorem 2.10] we can proceed as in the proof of Lemma 1.7 to get $\pi(U(A))=$ $U(A / I)^{\prime}$ and so (i) follows.

(ii) Since $U(A)$ is connected also is $\pi(U(A))$. As in the proof of Theorem 3.9 we can prove that $\pi(U(A))$ is clopen in $U(A / I)$, so $\pi(U(A))=U(A / I)^{0}$.

(iii) Notice that if $I=0$, then the result follows from [13, Proof of Theorem 2.10] or [16, Theorem 2.10]. Fix $n>1$. Since $A$ is purely infinite $A \approx M_{n}(A)$. By applying (i) to $\pi: M_{n}(A) \rightarrow M_{n}(A / I)$ we obtain $\pi\left(G E_{n}(A)\right)=\mathrm{GL}_{n}(A / I)^{\prime}$ and so $\mathrm{GL}_{n}(A / I)^{\prime} \subseteq G E_{n}(A / I)$.

Let $M \in \mathrm{GL}_{n}(A / I)$. Since $U(A / I) \rightarrow K_{1}(A / I)$ is onto, there exists a unit $u \in U(A / I)$ such that

$$
M\left(\begin{array}{cccc}
u & & & 0 \\
& 1 & & \\
0 & & & 1
\end{array}\right)=0 \in K_{1}(A / I) .
$$

But $K_{1}(A / I)=U(A / I)^{\text {ab }}$ implies

$$
M\left(\begin{array}{cccc}
u & & & 0 \\
& 1 & & \\
& & \ddots & \\
0 & & & 1
\end{array}\right) \in \mathrm{GL}_{n}(A / I)^{\prime},
$$

and by the above we have that $M \in G E_{n}(A / I)$ as desired.

THEOREM 3.14. Let $A$ be a purely infinite Rickart $C^{*}$-algebra satisfying general comparability. If $I$ is a closed ideal of $A$, then

(i) The map

$$
\alpha: \mathscr{F}(I, A) \rightarrow K_{0}(I), \quad x \mapsto\left[r\left(x^{*}\right)\right]-[r(x)]
$$

is a continuous monoid homomorphism which is onto that

(ii) $\left[r\left(x^{*}\right)\right]=[r(x)]$ if and only if there exists a projection $e \in I$ such

$$
r\left(x^{*}\right) \oplus e A \approx r(x) \oplus e A .
$$


(iii) $x, y \in \mathscr{F}(I, A)$ lie in the same connected component if and only if $\alpha(x)=\alpha(y)$. Furthermore $\alpha$ induces a group isomorphism

$$
K_{1}(A / I)=U(A / I) / U(A / I)^{0} \stackrel{\approx}{\rightarrow} K_{0}(I) .
$$

(iv) $\alpha(x)=0$ if and only if $x+I$ contains a unit.

Proof. By Proposition 3.7 (ii) we see that $\alpha$ is a well-defined monoid homomorphism. Since $A$ is purely infinite we have [13, Theorem 2.7 (ii) and the proof of Theorem 2.10] that $K_{1}(A)=0$. Clearly $K_{0}(A)=0$. Therefore the connecting map $\delta: K_{1}(A / I) \rightarrow K_{0}(I)$ is an isomorphism, in particular $\alpha$ is onto. By Theorem $3.13 K_{1}(A / I)=U(A / I) / U(A / I)^{0}$ so $\alpha$ is continuous. Thus we have shown (i) and a part of (iii). The remainder part of (iii) follows as in Theorem 3.9 (iv).

By Theorem 3.13, (iv) follows.

Now (ii) follows from Proposition 3.1.

If $A$ is an $A W^{*}$-algebra, then $A$ decomposes uniquely as a direct product $A_{1} \times A_{2}$ where $A_{1}$ is directly finite and $A_{2}$ is purely infinite. Now $A_{1}$ is a ring with stable range 1 so the connecting map associated with each ideal of $A_{1}$ is zero. Therefore we see that Theorem 3.14 is trivially true for $A_{1}$. Since any $A W^{*}$-algebra satisfies general comparability, Theorem 3.14 also holds for $A_{2}$. Thus we have

COROLlaRY 3.15. The conclusions of Theorem 3.14 are true for any closed ideal of an $A W^{*}$-algebra.

Finally we remark the following result which is an extension of Corollary 10.7 in [15] to $A W^{*}$-algebras.

Corollary 3.16. If I is an ideal of a $A W^{*}$-algebra $A$ of Type III, then every unit of $A / I$ can be lifted to a unit of $A$. If in addition $I$ is closed, then $U(A / I)$ is connected.

Proof. Let $\bar{I}$ be the closure of $I$ in $A$. Then since a unit in $A / \bar{I}$ lifts automatically to a unit of $A / I$, we may assume without loss of generality that $I$ is closed. Since $(e A)^{2} \approx e A$ for every idempotent $e$ in $I$ we see from Proposition 3.1 that $K_{0}(I)=0$. By Theorem 3.14 (iii) $U(A / I)=$ $U(A / I)^{0}$ is connected; and by Theorem 3.13 (i) we get $\pi(U(A))=$ $U(A / I)$. 
Acknowledgment. We would like to express our gratitude to Ken Goodearl for his contributions which led to improvements of an earlier version of this paper.

\section{REFERENCES}

[1] P. Ara and P. Menal, On regular rings with involution, Arch. Math., 42 (1984), 126-130.

[2] H. Bass, Algebraic K-theory, (Benjamin, New York, 1968).

[3] S. K. Berberian, Baer *-rings, Grundlehren Band 195 (Springer, Berlin and New York, 1972).

[4] M. Breuer, Fredholm theories in von-Neumann algebras I, Math. Ann., 178 (1968), 243-254.

[5] _ Fredholm theories in von-Neumann algebras II, Math. Ann., 180 (1969), 313-325.

[6] R. G. Douglas, Banach Algebra Techniques in Operator Theory, (Academic Press, New York and London, 1972).

[7] K. R. Goodearl, von Neumann Regular Rings, (Pitman, London, 1979).

[8] Directly finite aleph-nought-continuous regular rings, Pacific J. Math., 100, No. 1, (1982), 105-122.

[9] _ Notes on Real and Complex $C^{*}$-algebras, (Shiva Math. Series 5, 1982).

[10] K. R. Goodearl and P. Menal, preprint.

[11] D. Handelman, Finite Rickart $C^{*}$-algebras and their properties, Studies in Analysis Adv. in Math. Suppl. Studies, 4 (1979), 171-196.

[12] P. Menal and J. Moncasi, On regular rings with stable range 2, J. Pure and Appl. Algebra, 24 (1982), 25-40.

[13] „ $K_{1}$ of von Neumann regular rings, J. Pure and Appl. Algebra, 33 (1984), 295-312.

[14] J. Milnor, Introduction to algebraic K-theory, (Ann. of Math. Studies 72, Princeton, 1971).

[15] C. Olsen, Index theory in von Neumann algebras, (Memoirs of the Amer. Math. Soc., 294, 1984).

[16] M. A. Rieffel, Dimension and stable rank in the $K$-theory of $C^{*}$-algebras, Proc. London Math. Soc., (3), 46 (1983), 301-333.

[17] L. N. Vaserstein, Stable rank of rings and dimensionality of topological spaces, Funct. Anal. Appl., 5, 17-27 (translation 102-110) (1971).

[18] _ Bass's first stable range condition, J. Pure and Appl. Algebra, 34 (1984), 319-330.

[19] R. C. Walker, The Stone Cech Compactification, (Ergebnisse der Math. Band 83, Springer, Berlin and New York, 1974).

Received November 29, 1984 and in revised form December 10, 1985. This work has been supported by a grant from the Comisión Asesora de-Investigación Cientifica y Técnica. Ministerio de Educación y Ciéncia, Spain. 



\title{
PACIFIC JOURNAL OF MATHEMATICS \\ EDITORS
}

\section{S. VARADARAJAN}

(Managing Editor)

University of California

Los Angeles, CA 90024

HERBERT CLEMENS

University of Utah

Salt Lake City, UT 84112

R. FINN

Stanford University

Stanford, CA 94305
HERMANN FLASCHKA

University of Arizona

Tucson, AZ 85721

RAMESH A. GANGOLLI

University of Washington Seattle, WA 98195

VAughan F. R. Jones

University of California

Berkeley, CA 94720

ROBION KIRBY

University of California

Berkeley, CA 94720
C. C. MOORE

University of California

Berkeley, CA 94720

H. SAMELSON

Stanford University

Stanford, CA 94305

HAROLD STARK

University of California, San Diego

La Jolla, CA 92093

\section{ASSOCIATE EDITORS}

\author{
R. ARENS \\ E. F. BECKENBACH \\ B. H. NEUMANN \\ F. WOLF \\ K. YoshIDA \\ (1906-1982)

\section{SUPPORTING INS'TITUTIONS} \\ UNIVERSITY OF ARIZONA \\ UNIVERSITY OF BRITISH COLUMBIA \\ CALIFORNIA INSTITUTE OF TECHNOLOGY \\ UNIVERSITY OF CALIFORNIA \\ MONTANA STATE UNIVERSITY \\ UNIVERSITY OF NEVADA, RENO \\ NEW MEXICO STATE UNIVERSITY \\ OREGON STATE UNIVERSITY \\ UNIVERSITY OF OREGON \\ UNIVERSITY OF SOUTHERN CALIFORNIA \\ STANFORD UNIVERSITY \\ UNIVERSITY OF HAWAII \\ UNIVERSITY OF TOKYO \\ UNIVERSITY OF UTAH \\ WASHINGTON STATE UNIVERSITY \\ UNIVERSITY OF WASHINGTON
}

The Supporting Institutions listed above contribute to the cost of publication of this Journal, but they are not owners or publishers and have no responsibility for its content or policies.

Mathematical papers intended for publication in the Pacific Journal of Mathematics should be in typed form or offset-reproduced (not dittoed), double spaced with large margins. Please do not use built up fractions in the text of the manuscript. However, you may use them in the displayed equations. Underline Greek letters in red, German in green, and script in blue. The first paragraph must be capable of being used separately as a synopsis of the entire paper. In particular it should contain no bibliographic references. Please propose a heading for the odd numbered pages of less than 35 characters. Manuscripts, in triplicate, may be sent to any one of the editors. Please classify according to the scheme of Math. Reviews, Index to Vol. 39. Supply name and address of author to whom proofs should be sent. All other communications should be addressed to the managing editor, or Elaine Barth, University of California, Los Angeles, California 90024.

There are page-charges associated with articles appearing in the Pacific Journal of Mathematics. These charges are expected to be paid by the author's University, Government Agency or Company. If the author or authors do not have access to such Institutional support these charges are waived. Single authors will receive 50 free reprints; joint authors will receive a total of 100 free reprints. Additional copies may be obtained at cost in multiples of 50 .

The Pacific Journal of Mathematics is issued monthly as of January 1966. Regular subscription rate: $\$ 190.00$ a year (5 Vols., 10 issues). Special rate: $\$ 95.00$ a year to individual members of supporting institutions.

Subscriptions, orders for numbers issued in the last three calendar years, and changes of address should be sent to Pacific Journal of Mathematics, P.O. Box 969, Carmel Valley, CA 93924, U.S.A. Old back numbers obtainable from Kraus Periodicals Co., Route 100, Millwood, NY 10546.

The Pacific Journal of Mathematics at P.O. Box 969, Carmel Valley, CA 93924 (ISSN 0030-8730) publishes 5 volumes per year. Application to mail at Second-class postage rates is pending at Carmel Valley, California, and additional mailing offices. Postmaster: send address changes to Pacific Journal of Mathematics, P.O. Box 969, Carmel Valley, CA 93924.

PUBLISHED BY PACIFIC JOURNAL OF MATHEMATICS, A NON-PROFIT CORPORATION Copyright (c) 1987 by Pacific Journal of Mathematics 


\section{Pacific Journal of Mathematics}

\section{Vol. 126, No. 2 December, 1987}

Charles James Keith Batty, Derivations on the line and flows along orbits

Paul Erdốs, Adolf J. Hildebrand, Andrew Odlyzko, Paul Pudaite and

Bruce Reznick, The asymptotic behavior of a family of sequences . . . 227

Gregory James Galloway, A note on the fundamental group of a compact

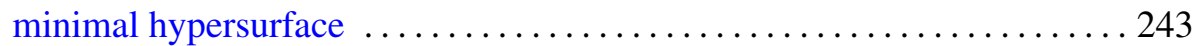

Nigel Higson, A characterization of $K K$-theory $\ldots \ldots \ldots \ldots \ldots \ldots \ldots \ldots 253$

Anthony To-Ming Lau and Wataru Takahashi, Weak convergence and nonlinear ergodic theorems for reversible semigroups of nonexpansive mappings

Pere Menal and Juame Moncasi, Lifting units in self-injective rings and an

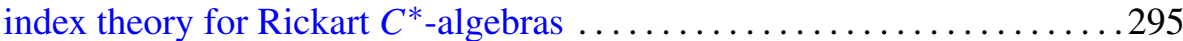

Yoshimi Saito, Schrödinger operators with a nonspherical radiation condition

Larry Smith, Realizing certain polynomial algebras as cohomology rings of

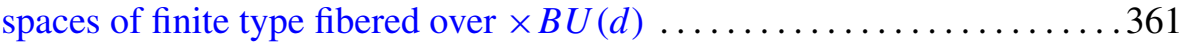

Carl E. Swenson and Calvin T. Long, Necessary and sufficient conditions for simple $A$-bases . . . . . . . . . . . . . . . . . . . . . . . . . . . . . . . 379

Kenneth Giovanni Valente, The $p$-primes of a commutative ring 385 